\title{
On the Relation Between Classical and Quantum Statistical Mechanics
}

\author{
W. Wreszinski * and G. Scharf \\ Institut für Theoretische Physik der Universität Zürich, Schoenberggasse 9, \\ CH-8001 Zürich, Switzerland
}

\begin{abstract}
Classical and quantum statistical mechanics are compared in the high temperature limit $\beta=1 / k T \rightarrow 0$. While this limit is rather trivial for spin systems, we obtain some rigorous results which suggest (and sometimes prove) different asymptotics for continuous systems, depending on the behaviour of the two-body potential for small distances: the difference between suitable classical and quantum variables vanishes as $\beta^{2}$ for smooth potentials and as $\sqrt{\beta}$ for potentials with hard cores.
\end{abstract}

\section{Introduction}

Although nature is governed by quantum mechanics, there is still much interest in classical statistical mechanics. This comes from the belief that for not too low temperatures the structural differences between the two are small. In fact, several deep similarities between classical and quantum lattice systems with regard to phase transitions are apparent [1]. Rigorous results on the low temperature behaviour of even the simplest quantum lattice systems from the point of view of ground state properties, namely, ferromagnetic quantum spin systems, are as yet incomplete [2]. For quantum continuous systems the situation is much worse, at least concerning rigorous results: very little is known on phase transitions for interacting systems, and low temperature properties are studied for just a few onedimensional models.

Another reason for interest in classical statistics is the fact that it is an approximation to quantum statistics in a precise sense. There is an extensive literature on the classical $\hbar \rightarrow 0$ limit of quantum statistics (see [3-5] and references given there). It is a weakness of these studies that no correction terms are provided. Then the limit $\hbar \rightarrow 0$ remains mathematical, because physically $\hbar$ cannot be varied. A reasonable statement for continuous systems would be: the difference between

\footnotetext{
* Supported in part by FAPESP. Permanent address: Instituto de Fisica, Universidade de Sao Paulo, Sao Paulo, Brazil
} 
quantum and classical quantities is small if the thermal wavelength $\lambda=\left(2 \pi \hbar^{2} \frac{\beta}{m}\right)^{1 / 2}$ is small with respect to a characteristic length of the potential. The physical parameter which is then actually varied is the (inverse) temperature $\beta \rightarrow 0$. For this interpretation of the $\hbar \rightarrow 0$ limit it is, of course, essential that it commutes with the thermodynamic limit. This causes difficulties, for example: the upper bound for the difference of the quantum $f^{\mathrm{Q}}(v, \beta)$ and classical $f^{\mathrm{Cl}}(v, \beta)$ canonical free energies (which we multiply by $\beta$ for reasons explained shortly),

$$
\Delta f(v, \beta)=\beta\left[f^{\mathrm{Q}}(v, \beta)-f^{\mathrm{Cl}}(v, \beta)\right],
$$

in [5] involves an attractive (temperature dependent) potential in the Bose case, which simulates the statistical exchange attraction, and taking the thermodynamic limit first requires, for reasons of stability, a singular repulsive (e.g. hard-core) potential. For such potentials, however, the lower bound of $[4,5]$ is not applicable, because it employs (plane-wave) eigenfunctions of the free Hamiltonian, which are not in the domain of the full Hamiltonian. Hence, at least with present methods, there is an essential gap in the classical $\hbar \rightarrow 0$ limit for bosons. For a class of bounded (and therefore, by stability, necessarily positive) potentials, the problem is solved by Simon's microstability condition [4].

In this paper we are primarily concerned with the physical form of the classical limit: $\beta \rightarrow 0$, keeping $\hbar$ fixed. For lattice systems, the high-temperature behaviour of $\Delta f$, defined by (I.1 a), follows trivially from Lieb's paper [3] on the classical limit of quantum spin systems (Appendix B). The corresponding result for continuous systems has been obtained only on a heuristic level using the Zassenhaus formula [14, Chap. 10.2]. A rigorous analysis shows that there is a marked difference in the high temperature asymptotics between bounded positive and hard core potentials. This can already be seen in the low-density region where the virial expansion is applicable. In Sect. 1 we present some estimates on the difference,

$$
\Delta b_{2}(\beta)=b_{2}^{\mathrm{Q}}(\beta)-b_{2}^{\mathrm{Cl}}(\beta),
$$

between the quantum and classical (direct) second-order virial coefficients [14], both for bounded positive and pure hard-core potentials. In the former case we show that

$$
\left|-\frac{\Delta b_{2}(\beta)}{b_{2}^{\mathrm{Cl}}(\beta)}\right| \underset{\beta \downarrow 0}{\leqq} c \beta .
$$

Throughout the paper, $c$ denotes a strictly positive constant, which may vary from inequality to inequality. The reason for considering $-\Delta b_{2}$, rather than $\Delta b_{2}$, in the estimates above and below, will be explained shortly. The exchange part of $\Delta b_{2}$ is known to decay exponentially as $\beta \downarrow 0[16,17]$, and does not, therefore, affect the leading behaviour above. For a pure hard-core potential we find (for $\beta$ sufficiently small),

$$
-c \sqrt{\beta} \leqq-\Delta b_{2}(\beta) \leqq 0
$$

with

$$
-\Delta b_{2}(\beta) \underset{\beta \downarrow 0}{\sim}-c \sqrt{\beta}, \quad v=1
$$


in dimension $v=1$. Indeed, on the basis of $[12,13]$, we expect

$$
-\Delta b_{2}(\beta) \underset{\beta \downarrow 0}{\sim}-c \sqrt{\beta} \quad(\text { all } v)
$$

in any dimension $v$, but the computations found in the literature (see, especially, [13]) rely on summation of various series involving phase-shifts $\delta_{\ell}(k)$ over all angular momenta (see also [14]). Indeed, for potentials with singularity of type $r^{-m}, m>2$ as $r \rightarrow 0$ ( $r$ denoting the radial distance from the origin), it is found that [15]

$$
\delta_{\ell}(k) \rightarrow A k^{1-2 / m}, k \rightarrow \infty, \ell \text { fixed } .
$$

As emphasized in [15], this conclusion leaves open the asymptotic behaviour of $\delta_{\ell}(k)$ as both $\ell$ and $k$ diverge. This is the main difficulty in obtaining rigorous estimates for $\Delta b_{2}(\beta)$ as $\beta \downarrow 0$ [which depend on the behaviour of all $\delta_{\ell}(k)$ for $k \rightarrow \infty$ ] by the phase-shift method. Nevertheless, the results of $[12,13]$ are believed to be exact. As a matter of fact, the dimensionality should not play a significant role as $\beta \downarrow 0$, affecting only the value of $c$ in (I.5). Therefore, we have not studied the problem for $v>1$. Indeed, the computed value of $c$ in (I.4) (Sect. II) is just one third of the coefficient in [13] for $v=3$.

The above results are consistent with the following asymptotic behaviour of $\Delta f(v, \beta)$ defined by $(\mathrm{I} .1 \mathrm{a})$,

$$
\begin{gathered}
\left|\frac{\Delta f(v, \beta)}{f^{\mathrm{Cl}}(v, \beta)}\right|_{\beta \downarrow 0} c \beta \quad \text { (positive bounded potential) }, \\
\frac{\Delta f(v, \beta)}{f^{\mathrm{Cl}}(v, \beta)} \underset{(\beta \downarrow 0)}{\cong}-c \sqrt{\beta} \quad \text { (hard-core system). }
\end{gathered}
$$

A nonrigorous, but probably asymptotically correct estimate suggests

$$
\frac{\Delta f(v, \beta)}{f^{\mathrm{Cl}}(v, \beta)}=+c \beta^{3}
$$

in (I.6a), see Sect. II. We first explain the correspondence between (I.2) and (I.6a), as well as (I.3) and (I.6b).

Denoting by $p^{\mathrm{Q}}(v, \beta)$ [respectively $\left.p^{\mathrm{Cl}}(v, \beta)\right]$ the canonical pressure, we have

$$
\beta\left(p^{\mathrm{Q}}(v, \beta)-p^{\mathrm{Cl}}(v, \beta)\right)=\left(\frac{\partial \Delta f(v, \beta)}{\partial v}\right)_{\beta}
$$

(as is well known $[19,21]$, this formula holds for almost all $v>v_{c}$, where $v_{c}$ is the close-packing specific volume). The virial expansion of the right-hand side of(I.7) is $[14,19]$

$$
\beta\left(p^{\mathrm{Q}}(v, \beta)-p^{\mathrm{Cl}}(v, \beta)\right)=\sum_{i \geqq 2} \frac{\Delta b_{i}(\beta)}{v^{i}},
$$

where $\Delta b_{i}(\beta)$ are differences between $i^{\text {th }}$ order quantum and classical virial coefficients, defined as in (I.1 b). By (I.7b) we find

$$
\Delta f(v, \beta)=\sum_{i \geqq 2} \frac{\Delta \tilde{b}_{i}(\beta)}{v^{i-1}},
$$


with

$$
\Delta \tilde{b}_{i}(\beta)=-i \Delta b_{i}(\beta)
$$

and hence

$$
\Delta \tilde{b}_{2}(\beta)=-2 \Delta b_{2}(\beta) .
$$

This explains the change of sign in the estimates (I.2), (I.3), and (I.4), to keep the correspondence with (I.6), as well as multiplication by $\beta$ in (I.1 a). that

A formal calculation using the Zassenhaus formula [14, Chap. 10.2] suggests

$$
\left|\Delta f(v, \beta) / f^{\mathrm{Cl}}(v, \beta)\right|_{\beta \downarrow 0}^{\sim} c \beta .
$$

This behaviour agrees with (I.6a) but not (I.6b). If the nonrigorous asymptotic estimate of Sect. II, Remark II.1, is true, the (I.7c) disagrees with the expected behaviour of both types of potential.

One might expect more than (I.6), for instance an asymptotic series in powers of $\sqrt{\beta}$ for hard core systems (in which case $f^{\mathrm{Cl}}$ is analytic in $\beta$ in a neighbourhood of $\beta=0$ [18]), eventually Borel-summable. This does not follow from the estimates in Ginibre's classic work (see [16] and references given there) and remains as the most interesting, but probably hardest, open question.

In Sect. III we derive some estimates for $\Delta f(v, \beta)$ for fixed finite, but arbitrary, density (smaller than close-packing). In (III.1) the upper bound of [5] is generalized to cover hard-core potentials, yielding the result that

$$
\Delta f(v, \beta) \leqq c \exp (-\alpha / \beta), c \geqq 0, \alpha>0 .
$$

For positive bounded pair potentials, it is easy to show by the methods of [5], that

$$
\lim _{\beta \downarrow 0} \Delta f(v, \beta)=0 \text { (bounded positive potentials) }
$$

in two cases (Fermi-Dirac and Maxwell-Boltzmann statistics). No correction term was obtained, so that (I.2) remains as the only precise indication of the validity of (I.6a). In III. 2 we derive a lower bound for $\Delta f(v, \beta)$ holding for a class of hard-core potentials. For a complete proof of (I.9) and correction terms, we need some information on the classical pure hard-core free energy $f_{\mathrm{Cl}}^{\text {h.c. }}(v)$, which is only available for $v=1$ and, in order not to clutter the argument with additional assumptions, we restrict ourselves to this case. It should be emphasized, however, that very similar, if not identical, arguments are expected to hold in higher dimensions. Indeed, in Sect. III.2.1 we provide, using methods of functional integration [11], a lower bound on the partition function for a class of hard-core systems which is useful in the thermodynamic limit and valid for all dimensions. As a corollary, we prove in Sect. III.2.2 that, if $v=1$,

$$
\Delta f(v, \beta) \geqq-c \beta^{\frac{1}{2}-\varepsilon}
$$

for sufficiently small $\beta$, and any $\varepsilon>0$. The reader will easily recognize what general properties of $f_{\mathrm{Cl}}^{\text {h.c. }}(v)$ are necessary for the validity of the proof of (III.2.2). Finally, we remark that even for pure hard cores and $v=1$ our result is nontrivial, because the one-dimensional quantum mechanical hard core problem is not solvable (for 
$a \neq 0$ ). In Sect. III.2.3 we show that Baumgartner's method [5] using the Thirring coherent states yield the weaker bound

$$
\Delta f(v, \beta) \geqq-c \beta^{1 / 3} .
$$

The above holds, however, for a class of interactions larger than the one we treat in (III.2.1).

Finally, in Appendix $\mathrm{C}$ we study the (equivalent) highenergy limit of the entropy, using the well-known method of Dirichlet-Neumann bracketing [10, Chap. XIII-15]. Although only bounded external fields are analysed there, the method is instructive, because, taking the thermodynamic limit first involves estimates of a completely different nature than those of [10, Chap. XIII, 15] for finite systems. It also yields correction terms of the expected type.

In Appendix A we collect some technical results used in the main text.

\section{High Temperature Behaviour of the Second Virial Coefficient}

In this section, we study the direct second order virial coefficient in two cases:

(A) for a positive infinitely differentiable potential of compact support $V \in C_{0}^{\infty}\left(\mathbb{R}^{3}\right)$;

(B) for a pure hard-core potential

$$
V(x)= \begin{cases}\infty & \text { if }|x| \leqq a \\ 0 & \text { otherwise }\end{cases}
$$

Since our aim is to obtain qualitative results, we did not try to determine the largest class of potentials for which the results of this section are expected to hold. For brevity, we refer to potentials of the above types as of class $\mathrm{A}$ and $\mathrm{B}$, respectively.

Case $(A)$. The classical second order virial coefficient $b_{2}^{\mathrm{Cl}}(\beta)$ is analytic in $\beta$ and

$$
b_{2}^{\mathrm{Cl}}(\beta)=\frac{1}{2} \int d^{3} x\left[1-e^{-\beta V(x)}\right] .
$$

The two-body quantum Hamiltonian is given on $L^{2}\left(\mathbb{R}^{3}\right)$ by

$$
\begin{aligned}
H & =H_{0}+V, \\
H_{0} & =-\frac{1}{2} \Delta
\end{aligned}
$$

( $h=m^{*}=1$, where $m^{*}=m / 2$ is the "reduced mass"). Let

$$
A(\beta) \equiv e^{-\beta H}-e^{-\beta H_{0}} .
$$

When $A(\beta)$ is trace-class, we shall assume that the thermodynamic limit of the direct quantum second-order virial coefficient per particle (see, e.g., [14] for the definitions in finite volume) exists independently of the boundary conditions and equals

$$
b_{2}^{\mathrm{Q}}(\beta)=-\frac{1}{2} \lambda^{3} \operatorname{tr} A(\beta)
$$

where

$$
\lambda=(2 \pi \beta)^{1 / 2}
$$


is the thermal wave-length. In case $(\mathrm{A}), A(\beta)$ is trace-class by [11, Theorem 21.4, p. 228], and for case (B) we shall comment later in the section. Let $d v_{a, c ; t}$ denote the probability measure formally associated to Wiener measure $d \mu_{0}$ by

$$
P_{t}(a, c)^{-1} \delta(\omega(0)-a) \delta(\omega(t)-c) d \mu_{0}
$$

(see definition, p. 39 of [11]), where $P_{t}(a, c)$ is defined below in (II.11); it corresponds to restriction to brownian paths $b(\cdot)$ [with $\omega(\cdot)=x+b(\cdot)]$, with preassigned positions at time zero and time $t$. Let $E_{0, a ; t, c}$ denote expectation with respect to $d v$ and $P_{0, a ; t, c}(A) \equiv E_{0, a ; t, c}\{\chi(A)\}$, where $\chi(A)$ denotes the characteristic function of the event $A$.

Proposition II.1. Let $V$ belong to class (A). Then

$$
\begin{aligned}
\Delta b_{2}(\beta) & =b_{2}^{\mathrm{Q}}(\beta)-b_{2}^{\mathrm{Cl}}(\beta) \\
& =\frac{1}{2} \int d^{3} x E_{0, x ; \beta, x}\left\{\exp [-\beta V(x)]-\exp \left[-\int_{0}^{\beta} d s V(\omega(s))\right]\right\} .
\end{aligned}
$$

Proof. Let

$$
f(x, \beta) \equiv E_{0, x ; \beta, x}\left\{\exp \left[-\beta V(x)-\exp \left[-\int_{0}^{\beta} d s V(\omega(s))\right]\right\} .\right.
$$

Suppose $x \notin \operatorname{supp} V$. Then, the only Brownian paths $b(\cdot)$ which contribute in the above expectation are those which start at $x$ and intersect $\operatorname{supp} V$ at least once before returning to $x$. As in [16], let

$$
\begin{aligned}
F^{\prime}(\beta, \varepsilon, \delta)= & \left\{b \mid \exists t \text { and } t^{\prime} \text { in }[0, \beta] \text { such that }\left|t-t^{\prime}\right| \leqq \delta,\right. \\
& \text { and } \left.\left|b(t)-b\left(t^{\prime}\right)\right|>4 \varepsilon\right\} .
\end{aligned}
$$

Clearly

$$
\begin{aligned}
F^{\prime}(\beta, \varepsilon, \beta) \supseteqq F^{\prime \prime}(\beta, \varepsilon) \equiv & \{b \mid \exists t \in[0, \beta] \text { such that } \\
& |b(t)-x|>4 \varepsilon\} .
\end{aligned}
$$

Setting $x=y$ in ((1.31) of [16, p. 343]), and taking into account the normalization in (II.6), we obtain

$$
P_{0, x ; \beta, x}\left(F^{\prime}(\beta, \varepsilon, \beta)\right) \leqq 16 \sqrt{2} \chi\left(\frac{\varepsilon}{2}, \beta\right),
$$

where

$$
\begin{gathered}
\chi(\varepsilon, \delta) \equiv \int_{|x|>\varepsilon} P_{\delta}(x, 0) d^{3} x, \\
P_{\delta}(x, y)=(2 \pi \delta)^{-3 / 2} \exp \left(-|x-y|^{2} / 2 \delta\right) .
\end{gathered}
$$

Hence, by (II.8), (II.9), (II.10) and the boundedness of $V$, there exist positive constants $c, d$, and $M$ such that

$$
\begin{aligned}
|f(x, \beta)| & \leqq c \exp (\beta M) P_{0, x ; \beta, x}\left(F^{\prime \prime}(\beta, d|x|)\right) \\
& \leqq 4 c \exp (\beta M) \chi\left(\frac{d|x|}{2}, \beta\right)
\end{aligned}
$$


which is exponentially decreasing in $|x|$ in view of (II.10). Hence, $f$ is integrable in $x$ for each $\beta$. Let $\left\{f_{n}\right\}_{n \in \mathbb{Z}_{+}}$be a sequence of functions in $C_{0}^{\infty}\left(\mathbb{R}^{3}\right)$ increasing monotonically to 1 . Then

$$
\frac{1}{2} \lambda^{3} \operatorname{tr}\left(f_{n} e^{-\beta H} f_{n}\right)=\frac{1}{2} \int d^{3} x f_{n}^{2}(x) E_{0, x ; \beta, x}\left\{\exp \left[-\int_{0}^{\beta} V(\omega(s)) d s\right]\right\}
$$

[11, Theorem 6.6], and similarly

$$
\frac{1}{2} \lambda^{3} \operatorname{tr}\left(f_{n} e^{-\beta H_{0}} f_{n}\right)=\frac{1}{2} \int d^{3} x f_{n}^{2}(x) .
$$

Let

$$
b_{2}^{\mathrm{Cl}}\left(\beta, f_{n}\right) \equiv \frac{1}{2} \int d^{3} x f_{n}(x)\left(1-e^{-\beta V(x)}\right) f_{n}(x) .
$$

Clearly,

$$
b_{2}^{\mathrm{Cl}}\left(\beta, f_{n}\right) \underset{n \rightarrow \infty}{\longrightarrow} b_{2}^{\mathrm{Cl}}(\beta) .
$$

Since $V \geqq 0$, [11, Theorem 21.4, p. 228] implies that $\operatorname{tr}\left(e^{-\beta H}-e^{-\beta H_{0}}\right)<\infty$. Hence, by Lemma A.1 of Appendix A,

$$
\operatorname{tr}\left(f_{n}\left(e^{-\beta H}-e^{-\beta H_{0}}\right) f_{n}\right) \underset{n \rightarrow \infty}{\longrightarrow} \operatorname{tr}\left(e^{-\beta H}-e^{-\beta H_{0}}\right),
$$

and therefore, by (II.4), (II.13), and (II.14),

$$
b_{2}^{\mathrm{Q}}(\beta)=\lim _{n \rightarrow \infty} \frac{1}{2} \int d^{3} x f_{n}^{2}(x) E_{0, x ; \beta, x}\left\{1-e^{-\int_{0}^{\beta} V(\omega(s)) d s}\right\} .
$$

By (II.15) and (II.16),

$$
\Delta b_{2}(\beta)=\frac{1}{2} \lim _{n \rightarrow \infty} \int d^{3} x f_{n}^{2}(x) E_{0, x ; \beta, x}\left\{e^{-\beta V(x)}-e^{-\int_{0}^{\beta} V(\omega(s))}\right\}^{d s} .
$$

By (II.12) the expectation occurring in (II.18) is uniformly bounded (in $x$ ) by an integrable function. Hence, (II.18) and the Lebesgue dominated convergence theorem yield (II.7).

Proposition II.2. Let $V$ belong to class (A). Then (I.2) holds.

Proof. The boundedness of $V$ and (II.7) allow us to write

$$
\begin{aligned}
\Delta b_{2}(\beta) & =\frac{1}{2} \int d^{3} x e^{-\beta V(x)} E_{0, x ; \beta, x}\left\{1-\exp \left[-\int_{0}^{\beta} d s(V(\omega(s))-V(x))\right]\right\} \\
& =-\frac{1}{2} \int d^{3} x e^{-\beta V(x)} E_{0, x ; \beta, x}\left\{\frac{\sum_{n=1}^{\infty}\left(-\int_{0}^{\beta} d s(V(\omega(s))-V(x))\right)}{n !}\right\} .
\end{aligned}
$$

Equation (II.19) may be justified in the same way as the steps leading to (II.18):

$$
\left|E_{0, x ; \beta, x}\left\{\frac{\sum_{n=1}^{\infty}\left(-\int_{0}^{\beta} d s V(\omega(s))-V(x)\right)^{n}}{n !}\right\}\right| \leqq c_{1} \chi\left(\frac{d_{1}|x|}{2}, \beta\right) e^{\beta M_{1}}
$$


for some positive constants $c_{1}, d_{1}, M_{1}$. Let, now, $\operatorname{supp} V \subseteq\{|x| \leqq a\}$. By (II.19)

$$
\Delta b_{2}(\beta)=I_{1}+I_{2},
$$

where

$$
I_{1} \equiv \frac{1}{2} \int_{|x| \leqq 2 a} d^{3} x e^{-\beta V(x)} E_{0, x ; \beta, x}\left\{\frac{\sum_{n=1}^{\infty}\left(-\int_{0}^{\beta} d s(V(\omega(s))-V(x))\right)^{n}}{n !}\right\},
$$

and

$$
I_{2} \equiv \frac{1}{2} \int_{|x|>2 a} d^{3} x E_{0, x ; \beta, x}\left\{1-\exp \left[-\int_{0}^{\beta} d s V(\omega(s))\right]\right\} .
$$

By the argument of Proposition II.1,

$$
\left|I_{2}\right| \leqq c \int_{|x|>2 a} d^{3} x \chi\left(d\left(\frac{|x|-a}{2}\right), \beta\right),
$$

where $\chi$ is defined by (II.10). For $\beta$ sufficiently small,

$$
\chi\left(\frac{d(|x|-a)}{2}, \beta\right) \leqq c \beta^{-1} \exp \left[-d^{2}(|x|-a)^{2} / 4 \beta\right] .
$$

The estimate above is not optimal but suffices for our purposes. Inserting (II.23) into (II.22) we see that

$$
\left|I_{2}\right| \leqq c \beta^{-1} \int_{r>2 a} d r r^{2} e^{-d^{2}(r-a)^{2} / 4 \beta},
$$

and therefore $I_{2}$ is exponentially small in $\beta^{-1}$, for $\beta$ sufficiently small. Hence, by (II.20), in order to establish (I.2), it suffices to prove that

$$
\left|I_{1}\right| \leqq c \beta^{2} .
$$

Let

$$
I_{1}^{\prime} \equiv \frac{1}{2} \int_{|x| \leqq 2 a} d^{3} x \exp [-\beta V(x)] E_{0, x ; \beta, x}\left\{\sum_{n=2}^{\infty}\left(-\int_{0}^{\beta} d s(V(\omega(s))-V(x))\right)^{n} / n !\right\} .
$$

By the boundedness of $V$,

$$
\left|I_{1}^{\prime}\right| \leqq c \beta^{2}
$$

Now,

$$
I_{1}=I_{1}^{\prime}+I_{2}^{\prime}
$$

where

$$
I_{2}^{\prime}=-\frac{1}{2} \int_{|x| \leqq 2 a} d^{3} x e^{-\beta V(x)} E_{0, x ; \beta, x}\left\{\int_{0}^{\beta} d s(V(\omega(s))-V(x))\right\} .
$$

We now use the explicit covariance formulae (see, e.g., [11, pp. 40, 41])

$$
\begin{gathered}
E_{0, x ; \beta, x}\left\{b_{i}(s)\right\}=0, \quad 0 \leqq s \leqq \beta, \\
E_{0, x ; \beta, x}\left\{b_{i}(s) b_{j}(t)\right\}=\delta_{i j}[s(\beta-t)], \quad 0 \leqq s \leqq t \leqq \beta,
\end{gathered}
$$


where $i, j=1,2,3$. In order to see the argument more clearly, consider the case $v=1$. Then

$$
V(x+b(s))-V(x)=\sum_{n=1}^{2 N-1} \frac{V^{(n)}(x)}{n !} b(s)^{n}+\frac{V^{(2 N)}(x+\theta b(s))}{(2 N) !} b(s)^{2 N},
$$

where $\theta \in[0,1]$ depends on $N$ and $b(s)$ and $V^{(n)}(x) \equiv \frac{d^{n} V(x)}{d x^{n}}$. In correspondence to (II.28c), let

$$
I_{2}^{\prime}=-\frac{1}{2} \int_{|x| \leqq 2 a} d^{3} x e^{-\beta V(x)}\left(\alpha_{1}(x)+\alpha_{2}(x)\right),
$$

where

$$
\alpha_{1}(x)=\sum_{n=1}^{2 N-1} \frac{V^{(n)}(x)}{n !} E_{0, x ; \beta, x}\left\{\int_{0}^{\beta} d s b(s)^{n}\right\},
$$

and

$$
\left|\alpha_{2}(x)\right| \leqq \frac{V^{(2 N)}(x+\theta b(s))}{(2 N) !} b(s)^{2 N} .
$$

By (II.28a, b), the Gaussian nature of the process and Wick's theorem (see, e.g., [11, Lemma 20.4, p. 217]),

$$
E_{0, x ; \beta, x}\left\{b(s)^{n}\right\}= \begin{cases}\frac{(2 m) !}{m ! 2^{m}} s^{m}(\beta-s)^{m} & \text { if } n=2 m \\ 0 & \text { otherwise. }\end{cases}
$$

We have

$$
\int_{0}^{\beta} d s s^{m}(\beta-s)^{m}=\beta^{2 m+1} B(m+1, m+1)=\frac{\beta^{2 m+1} m !^{2}}{(2 m+1) !} .
$$

Let

$$
G_{n} \equiv \sup _{x \in \mathbb{R}}\left|V^{(n)}(x)\right|
$$

Then, by (II.28e) and (II.29)

$$
\left|\alpha_{1}(x)\right| \leqq \sum_{m=1}^{N-1} \frac{G_{2 m} m !}{(2 m+1) ! 2^{m}} \beta^{2 m+1} \leqq c \beta^{3}
$$

for $\beta$ sufficiently small. By the same procedure applied to (II.28f),

$$
\left|\alpha_{2}(x)\right| \leqq \frac{\beta^{2 N+1} G_{2 N} N !}{(2 N+1) ! 2^{N}} .
$$

By (II.26), (II.27), (II.28d-f), (II.30a), and (II.30b), we obtain (II.25).

Remark II.1. Unfortunately, expansion of $V(x+b(s))-V(x)$ in each term of the series in (II.19) leads to a divergent, possibly asymptotic, series. Since (I.2) and (I.4) suffice to display the qualitative difference between potentials of classes (A) and (B), we do not pursue this question further here, but remark that (II.19) yields the 
following formal result. The leading contribution to (II.19) stems from

$$
\begin{aligned}
\Delta b_{2}(\beta)= & -\frac{1}{2} \int d^{3} x e^{-\beta V(x)} \frac{1}{2} \sum_{i=1}^{3}\left(\frac{\partial^{2} V}{\partial x_{i}^{2}}\right) \int_{0}^{\beta} d s E\left(b_{i}(s)^{2}\right) \\
& +\frac{1}{2} \int d^{3} x E_{0, x ; \beta, x}\left\{\frac{\left(\int_{0}^{\beta} d s\left(\sum_{i=1}^{3} \frac{\partial V}{\partial x_{i}}\right) b_{i}(s)\right)^{2}}{2}\right\} \\
\cong & -\frac{\beta^{3}}{24} \sum_{i=1}^{3} \int d^{3} x(1-\beta V(x)) \sum_{i=1}^{3}\left(\frac{\partial^{2} V}{\partial x_{i}^{2}}\right) \\
& +\frac{\beta^{4}}{24} \cdot \frac{1}{2} \int d^{3} x \sum_{i=1}^{3}\left(\frac{\partial V}{\partial x_{i}}\right)^{2}=-\frac{\beta^{4}}{48} \int d^{3} x \sum_{i=1}^{3}\left(\frac{\partial V}{\partial x_{i}}\right)^{2} .
\end{aligned}
$$

Notice that this term is strictly negative, in contrast with the sign in case (B), see below. If this result is asymptotically correct, and reflects the behaviour of $\Delta f(v, \beta)$, then, according to (I.6b), neither potentials of class (A) nor of class (B) agree with (I.7c).

Case (B). In this case (II.7) yields

$$
\Delta b_{2}(\beta)=\frac{1}{2} \int d^{3} x E_{0, x ; \beta, x}\{\chi(|x|>a)-\chi(|x+b(s)|>a ; 0 \leqq s \leqq \beta)\}
$$

(where $\chi$ is the characteristic function). The integrand above expresses the probability that the brownian motion during time $0 \leqq s \leqq \beta$ starting at $x$ (with $|x|>a)$ get into the ball of radius $a$. In the following, we present some results pertaining to $\Delta b_{2}(\beta)$.

Proposition II.3. Let $v=1$. Then

with $c=\frac{\sqrt{\pi}}{2 \sqrt{2}}$.

$$
-\Delta b_{2}(\beta) \underset{\beta \downarrow 0}{\sim}-c \sqrt{\beta}
$$

Proof. In case (B),

$$
b_{2}^{\mathrm{Cl}}(\beta)=a
$$

by (II.1) (if $v=1$ ). Quantum-mechanically, the corresponding Poisson kernel may be written down by the method of images

$$
P_{\beta}(x, y)=\frac{1}{\sqrt{2 \pi \beta}} \begin{cases}e^{-\frac{1}{2 \beta}(x-y)^{2}}-e^{-\frac{1}{2 \beta}(x+y-2 a)^{2}} & \text { for } x, y>a \\ e^{-\frac{1}{2 \beta}(x-y)^{2}}-e^{-\frac{1}{2 \beta}(x+y+2 a)^{2}} & \text { for } x, y<-a \\ 0 & \text { otherwise }\end{cases}
$$

This yields

$$
\begin{aligned}
\lambda \operatorname{tr}\left(e^{-\beta H}-e^{-\beta H_{0}}\right)= & \left\{-\int_{-a}^{a} d x-\int_{a}^{\infty} e^{-\frac{1}{2 \beta}(2 x-2 a)^{2}} d x\right. \\
& \left.-\int_{-\infty}^{-a} e^{-\frac{1}{2 \beta}(2 x+2 a)^{2}} d x\right\}=-\left(2 a+\sqrt{\frac{\pi \beta}{2}}\right),
\end{aligned}
$$

and hence by (II.32), we obtain (II.31). 
The three-dimensional result, believed to be exact, is [13]

$$
b_{2}^{\mathrm{Q}}(\beta)=b_{2}^{\mathrm{Cl}}(\beta)\left(1+\frac{\lambda}{a} \frac{3}{4}+\ldots\right) .
$$

Comparing with (II.31) and (II.32), we see that the correction to the classical result is expected to be proportional to $\sqrt{\beta}$, with a factor three times larger as the corresponding one in one dimension. In three-dimensions, a rigorous lower bound of the above form may be derived as follows. Let $H=H_{0}^{D}=-\frac{1}{2} \Delta^{D}$, with $\left(-\Delta^{D}\right)$ the Dirichlet Laplacian for the region $\mathbb{R}^{3} \backslash S$, where $S \equiv\left\{x \in \mathbb{R}^{3}:|x| \leqq a\right\}$, that is, the operator on $L^{2}\left(\mathbb{R}^{3} \backslash S\right)$ which is the Friedrichs extension of $(-\Delta)$ on $C_{0}^{\infty}\left(\mathbb{R}^{3} \backslash S\right)$. Let $\left(e^{-\beta H_{0}}-e^{-\beta H_{0}^{D}}\right)(x, y)$ denote the kernel of the operator $\left(e^{-\beta H_{0}}-e^{-\beta H_{0}^{D}}\right)$.

\section{Proposition II.4.}

$$
-c \sqrt{\beta} \leqq-\Delta b_{2}(\beta) \leqq 0
$$

for $\beta$ sufficiently small and $c>0$.

Proof. A nice and simple application of the functional method [11, Lemma 21.3, p. 227] yields

$$
0 \leqq\left(e^{-\beta H_{0}}-e^{-\beta H_{0}^{D}}\right)(x, y) \leqq(2 \pi \beta)^{-3 / 2} \exp \left\{-\frac{\left[\operatorname{dist}(x, S)^{2}+\operatorname{dist}(y, S)^{2}\right.}{4 \beta}\right\}
$$

if $x, y \in \Omega$, the unbounded component of $\mathbb{R}^{3} \backslash S$. It is proved (although not formally stated there) in Theorem 21.4, p. 228, of [11] that $\left(e^{-\beta H_{0}}-e^{-\beta H_{0}^{D}}\right)$ is trace class. We have then

$$
\begin{aligned}
b_{2}^{\mathrm{Q}}(\beta) & =-\frac{1}{2} \lambda^{3} \operatorname{tr}\left(e^{-\beta H_{0}^{D}}-e^{-\beta H_{0}}\right) \\
& =\frac{1}{2}(2 \pi \beta)^{3 / 2} \operatorname{tr}\left(e^{-\beta H_{0}}-e^{-\beta H_{0}^{D}}\right) .
\end{aligned}
$$

From (II.1),

$$
b_{2}^{\mathrm{Cl}}(\beta)=\frac{2}{3} \pi a^{3} .
$$

By (II.34), (II.35), and (II.36):

$$
\begin{aligned}
b_{2}^{\mathrm{Q}}(\beta)= & \frac{1}{2}(2 \pi \beta)^{3 / 2}\left\{\int_{|x| \leqq a} d^{3} x\left(e^{-\beta H_{0}}\right)(x, x)\right. \\
& \left.+\int_{|x| \geqq a} d^{3} x\left[e^{-\beta H_{0}}(x, x)-\left(e^{-\beta H_{0}^{\mathrm{D}}}\right)(x, x)\right]\right\} \\
\leqq & b_{2}^{\mathrm{Cl}}(\beta)+\frac{4 \pi}{2} \int_{a}^{\infty} d r r^{2} e^{-(r-a)^{2} / 2 \beta} \\
= & b_{2}^{\mathrm{Cl}}(\beta)+c_{1} \sqrt{\beta}+c_{2} \beta+c_{3} \beta^{3 / 2},
\end{aligned}
$$

with $c_{1}, c_{2}$, and $c_{3}$ strictly positive constants. This implies (II.33).

Notice that (II.31) and (II.33) are, respectively, (I.4) and (I.3) of the introduction.

As a final remark, it is an open problem to prove that the estimates in the present section yield the corresponding estimates (I.6) for the free energy even for 
sufficiently small density by the methods of [16], except in case (A). In the latter case, (I.6a) follows rigorously from Proposition II.2 or II.3 if the density is sufficiently small [16]. We are, however, interested in fixed (but arbitrary) density and $\beta$ sufficiently small. This motivated our search for direct bounds on the free energy in the next sections.

\section{Upper and Lower Bounds for $\boldsymbol{\Delta} f(\boldsymbol{v}, \beta)$}

\section{III.1. Upper Bound for $\Delta f(v, \beta)$}

In this section we generalize the upper bound of [5] to hard-core systems. We consider $N$ particles in a $v$-dimensional hypercube $\Lambda^{v}$ and the corresponding Hilbert space $\mathscr{H}_{\Lambda}^{(N)}=L^{2}\left(\Lambda^{v N}\right)$. To each particle is associated a momentum $p_{i}$, $i=1, \ldots, N$, which acts as a differential operator on $\mathscr{H}_{\Lambda}^{(N)}$ :

$$
\left(p_{j} \psi\right)\left(x_{1}, \ldots, x_{N}\right)=-i \hbar \nabla_{x} \psi\left(x_{1}, \ldots, x_{N}\right)
$$

where $D\left(p_{j}\right)=C_{0}^{\infty}\left(\Lambda^{v N}\right)$. Define the free Dirichlet Hamiltonian (kinetic energy) as the self-adjoint operator $H_{0, \Lambda}$ associated with the closure of the quadratic form

$$
h_{0}(\psi)=\sum_{i=1}^{N}\left\|p_{i} \psi\right\|^{2}, D\left(h_{0}\right)=C_{0}^{\infty}\left(\Lambda^{v N}\right) .
$$

The Hilbert space $\mathscr{H}_{\Lambda, a}^{(N)}$ for $N$ particles with spherical hard cores of diameter $a$ is a closed subspace of $\mathscr{H}_{\Lambda}^{(N)}$ spanned by $\psi \in \mathscr{H}_{\Lambda}^{(N)}$ which vanish on the set

$$
S_{a}^{(N)}=\left\{\left(x_{1}, \ldots, x_{N}\right) ; x_{i} \in \mathbb{R}^{v},\left|x_{i}-x_{j}\right| \leqq a \text { for some } i \neq j \in\{1,2, \ldots, N\}\right\} .
$$

We now define the free energy for hard-core systems $H_{0, \Lambda}^{a}$ as the (positive, selfadjoint) operator corresponding to the Friedrichs extension associated to the positive quadratic form

$$
h_{0}^{a}(\psi)=\sum_{i=1}^{N}\left\|p_{i} \psi\right\|^{2},\left(h_{0}^{a}\right)=C_{0}^{\infty}\left(\Lambda^{v N} \backslash S_{a}^{(N)}\right) .
$$

In order to extend the upper bound of [5] to hard-core systems, some problems arise. The main proof in [5] is valid only on the whole Hilbert space $\mathscr{H}_{A}^{(N)}$. Let $V$ be an interparticle interaction, given as a multiplication by a real potential:

$$
(V \psi)\left(x_{1}, \ldots, x_{N}\right)=v\left(x_{1}, \ldots, x_{N}\right) \psi\left(x_{1}, \ldots, x_{N}\right)
$$

We assume that

$$
v \in L^{\infty}\left(\Lambda^{N v}\right)
$$

Under the above assumption, the Friedrichs extension and the form sum extension of $\left(H_{0, \Lambda}^{a}+V\right)$ are equal. Let, now, $\left\{U_{m}\right\}_{m \geqq 0}$ be a set of interactions on $\mathscr{H}_{\Lambda}^{(N)}$ defined by the potentials

$$
U_{m}\left(x_{1}, \ldots, x_{N}\right)=\left\{\begin{array}{lll}
m & \text { if } & \left(x_{1}, \ldots, x_{N}\right) \in S_{a}^{(N)} \\
0 & \text { if } & \left(x_{1}, \ldots, x_{N}\right) \notin S_{a}^{(N)}
\end{array} .\right.
$$

Under the above assumptions on $V$, it follows from Lemma A.2 of Appendix A that $H_{0, \Lambda}+U_{m}+V$ is self-adjoint on $D\left(H_{0, \Lambda}\right)$ and converges to the Friedrichs extension $H_{\Lambda}^{a}$ of $H_{0, \Lambda}^{a}+V$ in the strong resolvent sense as $m \rightarrow \infty$. 
Explicitly,

$$
\begin{gathered}
\lim _{m \rightarrow \infty}\left\|\left[\left(H_{0, \Lambda}+U_{m}+V+E\right)^{-1}-\left(H_{A}^{a}+E\right)^{-1}\right] \psi\right\|=0, \psi \in \mathscr{H}_{\Lambda, a}^{(N)}, \\
\lim _{m \rightarrow \infty}\left\|\left(H_{0, \Lambda}+U_{m}+V+E\right)^{-1} \psi\right\|=0, \psi \in \mathscr{H}_{\Lambda, a}^{(N) \perp},
\end{gathered}
$$

for all $E>0$ (if $V \geqq 0$ - otherwise add a constant to $V$ ). We are now able to state the main result of this section. Let $Z_{\Lambda, \mathrm{B}}(\beta), Z_{A, \mathrm{~F}}(\beta)$, and $Z_{\Lambda, \mathrm{MB}}(\beta)$ denote the canonical partition functions for Bose-Einstein, Fermi-Dirac, and Maxwell-Boltzmann statistics, defined in the usual way [19] for hard-core systems satisfying the assumptions stated above.

Theorem III.1. a) $Z_{\Lambda, \mathrm{B}}(\beta) \leqq \tilde{Z}_{\Lambda}^{\mathrm{Cl}}(\beta)$,

b) $Z_{\Lambda, \mathrm{F}}(\beta) \leqq Z_{\Lambda}^{\mathrm{Cl}}(\beta)$,

c) $Z_{\Lambda, \mathrm{MB}}(\beta) \leqq Z_{\Lambda}^{\mathrm{Cl}}(\beta)$,

where

$$
Z_{\Lambda}^{\mathrm{Cl}}(\beta)=\frac{1}{N !} \int_{x \in \Lambda^{N \nu}\left(S_{c}^{N)}\right.} \frac{d^{N v} x d^{N v} p}{(2 \pi \hbar)^{N v}} \exp \left[-\beta H_{\Lambda}^{\mathrm{Cl}}(p, x)\right],
$$

with

$$
H_{\Lambda}^{\mathrm{Cl}}(p, x)=\sum_{i=1}^{N} p_{i}^{2}+v\left(x_{1}, \ldots, x_{N}\right)
$$

is the classical partition function, and $\tilde{Z}_{\Lambda}^{\mathrm{Cl}}$ is the classical partition function corresponding to the Hamiltonian

$$
\begin{gathered}
\tilde{H}_{A}^{\mathrm{Cl}}(p, x)=H_{\Lambda}^{\mathrm{Cl}}(p, x)+v_{B}\left(x_{1}, \ldots, x_{N}\right), \\
v_{B}\left(x_{1}, \ldots, x_{N}\right)=\sum_{1 \leqq i<j \leqq N} v_{B}\left(\left|x_{i}-x_{j}\right|\right), \\
\tilde{v}_{B}(|x|)=-\frac{1}{\beta} \exp \left(-\frac{1}{4 \beta \hbar^{2}}|x|^{2}\right) .
\end{gathered}
$$

(Note a sign misprint in (30) of [5].) As in [5], we took $2 m=1$ for simplicity). Proof. Let $T_{\beta}$ be defined on $\mathscr{H}_{A}^{(N)}$ by

$$
T_{\beta} \psi=\left\{\begin{array}{lll}
e^{-\beta H_{\Lambda}^{0}} \psi & \text { if } & \psi \in \mathscr{H}_{\Lambda, a}^{(N)} \\
0 & \text { if } & \psi \in \mathscr{H}_{\Lambda, a}^{(N)^{\perp}},(\beta>0)
\end{array} .\right.
$$

By (III.8), (III.14), and Lemma A.3 of Appendix A,

$$
\exp \left[-\beta\left(H_{0, \Lambda}+U_{m}+V\right)\right] \psi \underset{m \rightarrow \infty}{\longrightarrow} T_{\beta} \psi \forall \psi \in \mathscr{H}_{\Lambda}^{(N)} .
$$

By (III.15) and Proposition A.1 of Appendix A:

$$
\begin{aligned}
Z_{A, \mathrm{~B}}(\beta) & =\operatorname{tr}_{\mathscr{H}}^{(N), a} P_{\beta} \exp \left(-\beta H_{A}^{a}\right)=\operatorname{tr}_{\mathscr{H}(\Lambda)}\left(P_{B} T_{\beta}\right) \\
& =\lim _{m \rightarrow \infty} \operatorname{tr}_{\mathscr{H}(N)} P_{B} \exp \left[-\beta\left(H_{0, A}+U_{m}+V\right)\right] .
\end{aligned}
$$


As in [5], $P_{B}$ denotes the projector onto the symmetric (Boson) subspace. We may now follow the proof of [5, p. 33], based on the Golden-Thompson inequality, to find:

$$
\begin{aligned}
& \operatorname{tr}_{\mathscr{H}(N)} P_{B} \exp \left[-\beta\left(H_{0}+U_{m}+V\right)\right] \\
& \leqq \\
& \quad \int \frac{d^{N v} x d^{N v} p}{(2 \pi \hbar)^{N v}} \exp \left[-\beta\left(\sum_{i=1}^{N} p_{i}^{2}+U_{m}\left(x_{1}, \ldots, x_{N}\right)\right.\right. \\
& \left.\left.\quad+v\left(x_{1}, \ldots, x_{N}\right)+v_{B}\left(x_{1}, \ldots, x_{N}\right)\right)\right]
\end{aligned}
$$

Taking now the limit of the right-hand side of (III.17), as $m \rightarrow \infty$, and using the monotone convergence theorem, we finally obtain a). The proofs of b) and c) are similar.

In order to prove (I.8), let $V_{A}$ be the volume of $\Lambda$ and define the "free energies":

$$
f_{\Lambda, \mathrm{B}}^{\mathrm{Q}}(\beta)=\frac{1}{V_{A}} \log Z_{\Lambda, \mathrm{B}}(\beta)
$$

with similar definitions for $f_{\Lambda, \mathrm{F}}^{\mathrm{Q}}(\beta)$ and $f_{\Lambda, \mathrm{MB}}^{\mathrm{Q}}(\beta)$, and

$$
f_{A}^{\mathrm{Cl}}(\beta)=\frac{1}{V_{A}} \log Z_{\Lambda}^{\mathrm{Cl}}(\beta) .
$$

We also denote the thermodynamic limit $[19,21]$ of $f_{\Lambda}(\beta)$ by $f(v, \beta)$, as in Sect. I, where $v=\lim _{\left(V_{A}, N \rightarrow \infty\right)} \frac{V_{A}}{N}$ is the specific volume. Note that by (III.18) our $f(v, \beta)$ differs from the usual canonical free energy by a factor $(-\beta)$, conforming to the definition (I.1) already discussed in that section.

Theorem III.2. Under the assumptions of this section for hard-core systems, (I.8) holds.

Proof. According to b) and c) of Theorem III.1, (I.8) holds for Fermi-Dirac and Maxwell-Boltzmann statistics, with $c=0$. We now concentrate on the Bose case. We have, by (III.18), (III.12) and a) of Theorem III.1,

$$
\begin{aligned}
f_{A, \mathrm{~B}}^{\mathrm{Q}}(\beta)-f_{A}^{\mathrm{Cl}}(\beta) & =\frac{1}{V_{A}} \log \frac{Z_{\Lambda, \mathrm{B}}(\beta)}{Z_{\Lambda}^{\mathrm{Cl}}(\beta)} \\
& \leqq \varrho \sup _{N} \sup _{\substack{x_{i} \in \mathbb{R}^{v} \\
i=1, \ldots, N \\
\left|x_{i}-x_{j}\right| \geqq a, i \neq j}}\left[\frac{\left|\beta v_{B}\left(x_{1}, \ldots, x_{N}\right)\right|}{N}\right],
\end{aligned}
$$

where $\varrho=\frac{1}{v}$ is the density. Now, by [21, Appendix A], and (III.13),

$$
\sup _{\substack{x_{i} \in \mathbb{R},, i=1, \ldots, N \\\left|x_{i}-x_{j}\right| \geqq a, i \neq j}}\left|\beta v_{B}\left(x_{1}, \ldots, x_{N}\right)\right| \leqq \beta B N
$$


where

$$
\beta B=\sum_{t \neq 0} \sup _{x \in \Delta(t)}\left|\beta \tilde{v}_{B}(x)\right|
$$
and the sum is over all cubic cells $\Delta(t)$ centered at the points $t=n d$, with $d=\frac{a}{\sqrt{v}}$ and
$n \in \mathbb{Z}^{v}$. Let

$$
f(\ell)=\exp -\left[\frac{1}{4 \beta \hbar^{2}}\left(\ell-\frac{1}{2}\right)^{2} d^{2}\right], \quad \ell=1,2,3, \ldots
$$

Then, by (III.20b) and (III.21), and setting $v=3$ :

$$
\beta B \leqq 3.2 \sum_{\ell=1}^{\infty} f(\ell)+3.4\left(\sum_{\ell=1}^{\infty} f(\ell)\right)^{2}+8\left(\sum_{\ell=1}^{\infty} f(\ell)\right)^{3} .
$$

Above, we considered separately the cells centered at points on the three coordinate axes, as well as those centered at points on the three coordinate planes, and disregarded double or multiple countings. A similar estimate holds for general v. Equation (I.8) follows immediately from [(III.19)-(III.22)] and the Euler summation formula (see, e.g., [22, Appendix]) applied to the function $f$.

Remark III.1. The constant $\alpha$ in (I.8) may be evaluated

$$
\alpha=\left(\frac{d}{8 \hbar}\right)^{2}=\left(\frac{a}{8 \sqrt{3} \hbar}\right)^{2}
$$

The thermal wavelength is $\lambda=\sqrt{\pi \beta} \hbar$. Hence the right-hand side of (I.8) is exponentially small in the ratio $a / \lambda$, a precise version of the condition $v^{1 / 3}(>a) \gg \lambda$ [14, Chap. 10.2].

\section{III.2. Lower Bound for Hard-Core System}

III.2.1. Lower Bound for the Partition Function. In this section we derive a lower bound for the partition function, using methods of functional integration $[11,16]$. We assume a positive bounded potential $v$ in (III.5) in addition to a hard core of diameter $a$, satisfying

$$
\begin{gathered}
v\left(x_{1}, \ldots, x_{N}\right)=\sum_{1 \leqq i<j \leqq N} \tilde{v}\left(\left|x_{i}-x_{j}\right|\right), \\
\tilde{v}(|x|)=0 \quad \text { if } \quad|x|<a \\
0 \leqq \tilde{v}(|x|) \quad \forall x \in \mathbb{R}^{v} .
\end{gathered}
$$

For some $\varepsilon>0$ and

$$
\begin{gathered}
0<D<\infty, \tilde{v}(|x|) \leqq D /(1+|x|)^{v+\varepsilon} \forall x \in \mathbb{R}^{v}, \\
\tilde{v}(\lambda|x|) \leqq \tilde{v}(|x|) \lambda \geqq 1 \quad \forall x \in \mathbb{R}^{v} .
\end{gathered}
$$

Condition (III.26) suffices for stability [21]. Assumption (III.27) of monotone decrease is, as we shall see, essential. We first approximate the full Hamiltonian by $\left(H_{0, \Lambda}+U_{m}+V\right)$ on $\mathscr{H}_{\Lambda}(N)$ as in Sect. III.1. 
We choose $U_{m}$ of the form:

$$
U_{m}\left(x_{1}, \ldots, x_{N}\right)=\sum_{1 \leqq i<j \leqq N} u_{m}\left(\left|x_{i}-x_{j}\right|\right),
$$

where

$$
u_{m}(|x|)=\left\{\begin{array}{ll}
m & \text { if }|x|<a \\
0 & \text { otherwise }
\end{array} .\right.
$$

Although $U_{m}$ is not of the form (III.7), a modification of Lemma A.3 and the proof in Sect. III.1 applies to yield (in this section we consider for simplicity Maxwell Boltzmann statistics only)

$$
\begin{gathered}
Z_{\Lambda, \mathrm{MB}}(\beta)=\lim _{m \rightarrow \infty} Z_{\Lambda}^{(m)}(\beta), \\
Z_{\Lambda}^{(m)}(\beta)=\frac{1}{N !} \operatorname{tr}_{\mathscr{H}\left({ }_{\Lambda}^{(N)}\right)} e^{-\beta\left(H_{0, \Lambda}+U_{m}+V\right)} .
\end{gathered}
$$

We write

$$
U_{m}+V=\sum_{1 \leqq i<j \leqq N} w_{m}\left(\left|x_{i}-x_{j}\right|\right)
$$

where

$$
w_{m}(|x|)=u_{m}(|x|)+\tilde{v}(|x|),
$$

and choose $m$ so large such that $w_{m}$ also satisfies (III.27). Let $d x$ denote as usual Lebesgue measure on $\mathbb{R}^{v}$ and let $(B, \beta, D b)$ be the measure space for $v$-dimensional Brownian motion $[11$, p. 38]. We follow the same notation of [11] except for denoting vectors by a special (bold-faced) symbol. As in [11, p. 38], define Wiener measure as the measure $d \mu_{0}$ on $\mathbb{R}^{v} \times B$ given by $d x \otimes D b$, and let $\omega(t)=x+b(t)$. As in $\left[11\right.$, p. 39], let $d \mu_{0, a, c}(\omega)$ denote conditional Wiener measure [which corresponds formally, to setting $\omega(0)=a$, and $\omega(t)=c]$. Let

$$
\chi_{\Lambda}(\omega)= \begin{cases}1 & \text { if } \omega(t) \in \Lambda \text { for all } t \in[0, \beta] \\ 0 & \text { otherwise }\end{cases}
$$

Then [23, Theorem 6.3.9] (conditional) Wiener measure corresponding to Dirichlet boundary conditions on $\Lambda$ is obtained from the free measure $d \mu_{0, a, c ; t}$ by multiplication with the characteristic function $\chi_{A}$ of $\Lambda$. In particular, let $\{\alpha(s)\}_{0 \leqq s \leqq 1}$ denote Brownian bridge $[11$, p. 40], related to $\omega$ by $\omega(t)$ $=x+\sqrt{\beta} \alpha(t / \beta)$. Then the Feynman-Kac formula, together with (4.13) of [11, Chap. II.4], yields the following representation for $Z_{\Lambda}^{(m)}(\beta)$ in (III.29a):

$$
\begin{aligned}
& Z_{\Lambda}^{(m)}(\beta)=\frac{1}{N !}(2 \pi \beta)^{-v N / 2} \int_{\Lambda^{N \nu}} d x \int D \alpha_{1}, \ldots, D \alpha_{N} \exp \left\{-\int_{0}^{\beta} d t \sum_{1 \leqq i<j \leqq N} w_{m}\left(\mid x_{i}-x_{j}\right.\right. \\
& \left.\left.\quad+\sqrt{\beta}\left[\alpha_{i}\left(\frac{t}{\beta}\right)-\alpha_{j}\left(\frac{t}{\beta}\right)\right] \mid\right)\right\} \chi_{\Lambda}\left(x_{1}+\sqrt{\beta} \alpha_{1}\left(\frac{t}{\beta}\right)\right) \times \ldots \times \chi_{\Lambda}\left(x_{N}+\sqrt{\beta} \alpha_{N}\left(\frac{t}{\beta}\right)\right) .
\end{aligned}
$$


Above, $D \alpha_{i}(i=1, \ldots, N)$ is a copy of the measure corresponding to Brownian bridge. Notice that the trace corresponds to setting $a=c$ in (4.13) of [11].

Let

$$
\varrho_{i}(t)=\sqrt{\beta} \alpha_{i}\left(\frac{t}{\beta}\right)
$$

If $\left|\varrho_{i}(t)-\varrho_{j}(t)\right| \leqq \delta\left|x_{i}-x_{j}\right|$, then $(\delta<1)$,

$$
\left|x_{i}-x_{j}+\varrho_{i}(t)-\varrho_{j}(t)\right| \geqq(1-\delta)\left|x_{i}-x_{j}\right|,
$$

and by the monotone-decrease property of $w_{m}$ :

$$
w_{m}\left(\left|x_{i}-x_{j}+\varrho_{i}(t)-\varrho_{j}(t)\right|\right) \leqq w_{m}\left[(1-\delta)\left|x_{i}-x_{j}\right|\right] .
$$

Notice that if $A, B, C$ denote the events

$$
\begin{aligned}
A= & \left\{\sup _{0 \leqq s \leqq 1}\left|\alpha_{i}(s)-\alpha_{j}(s)\right| \leqq \frac{\delta}{\sqrt{\beta}}\left|x_{i}-x_{j}\right| ; \forall i, j=1, \ldots, N, i \neq j ;\right. \\
& \left.x_{i}+\sqrt{\beta} \alpha_{i}(s) \in \Lambda^{v}, \forall i=1, \ldots, N, \forall s \in[0,1]\right\}, \\
B= & \left\{\sup _{0 \leqq s \leqq 1}\left|\alpha_{i}^{(k)}(s)-\alpha_{j}^{(k)}(s)\right| \leqq \frac{\delta}{\sqrt{v} \sqrt{\beta}}\left|x_{i}-x_{j}\right| ; \forall i, j=1, \ldots, N ;\right. \\
& \left.i \neq j, \forall k=1, \ldots, v ; x_{i}+\sqrt{\beta} \alpha_{i}(s) \in \Lambda^{v}, \forall i=1, \ldots, N, \forall s \in[0,1]\right\}, \\
C= & \left\{\begin{array}{c}
\sup _{i \in[1, N]} \sup _{k \in[1, v]} \leqq s \leqq 1 \\
\end{array}\right. \\
& \left.x_{i}+\sqrt{\beta} \alpha_{i}^{(k)}(s)\left|\leqq \frac{\delta}{2 \sqrt{v} \sqrt{\beta}}\right| x_{i}(s) \in \Lambda^{v}, \forall i=1, \ldots, N, s \in[0,1]\right\}, \forall i, j=1, \ldots, N, i \neq j ;
\end{aligned}
$$

then $A \supseteqq B \supseteqq C$. Now, by (III.31) and (III.32):

$Z_{\Lambda}^{(m)}(\beta)$

$$
\geqq \frac{1}{N !}(2 \pi \beta)^{-v N / 2} \int_{\Lambda^{N v}} d x_{1} \ldots d x_{N} \exp \left\{-\beta \sum_{1 \leqq i<j \leqq N} w_{m}\left[(1-\delta)\left|x_{i}-x_{j}\right|\right]\right\} \cdot E_{N}(A),
$$

where $E_{N}(\cdot)$ denotes expectation with respect to the measure $D \alpha_{1} \ldots D \alpha_{N}$. Taking now the limit $m \rightarrow \infty$ on (III.33) and using the monotone convergence theorem, we obtain by (III.29a) and $A \supseteqq C$ :

$$
Z_{\Lambda, \mathrm{MB}}(\beta) \geqq \frac{1}{N !}(2 \pi \beta)^{-N v} \int_{\Lambda^{v N \backslash S\left(a_{1}^{N}\right)}} d x_{1} \ldots d x_{N} \exp \left\{-\beta \sum_{1 \leqq i<j \leqq N} \tilde{v}\left(\left|x_{i}-x_{j}\right|\right)\right\} \cdot E_{N}(C),
$$

where

$$
a_{1} \equiv \frac{a}{1-\delta}
$$


Now, given that $\left(x_{1}, \ldots, x_{N}\right) \notin S_{a_{1}}^{(N)}$, then $C \supseteqq D$, where

$$
\begin{aligned}
D= & \left\{\sup _{0 \leqq s \leqq 1}\left|\alpha_{i}^{(k)}(s)\right| \leqq \frac{\delta a_{1}}{2 \sqrt{v} \sqrt{\beta}}, \forall i=1, \ldots, N, \forall k=1, \ldots, v ;\right. \\
& \left.x_{i}+\sqrt{\beta} \alpha_{i}(s) \in \Lambda^{v}, \forall i=1, \ldots, N, \forall s \in[0,1]\right\} .
\end{aligned}
$$

Let

$$
\Lambda^{\prime v} \equiv\left\{x \in \Lambda^{v} ;\left|x^{(k)}\right| \leqq \ell-\frac{\delta a_{1}}{2 \sqrt{v}}, k=1, \ldots, v\right\}
$$
where $\Lambda^{v}=[-\ell, \ell]^{v}$. Then $x \in \Lambda^{\prime v}$ and $\left|\alpha^{(k)}(s)\right| \leqq \frac{\delta a_{1}}{2 \sqrt{v} \sqrt{\beta}}, \forall s \in[0,1]$, imply
$(x+\sqrt{\beta} \alpha(s)) \in \Lambda^{v}$.

Therefore, given that $x \in\left(\Lambda^{\prime}\right)^{v N} \backslash S_{a_{1}}^{(N)}$, it follows that $D \supseteqq F$, where

$$
F \equiv\left\{\sup _{0 \leqq s \leqq 1}\left|\alpha_{i}^{(k)}(s)\right| \leqq \frac{\delta a_{1}}{2 \sqrt{v} \sqrt{\beta}}, \quad \forall i=1, \ldots, N, \forall k=1, \ldots, v\right\} .
$$

Hence, we finally get

$$
\begin{gathered}
Z_{\Lambda, \mathrm{MB}}(\beta) \geqq \frac{1}{N !}(2 \pi \beta)^{-v N / 2} \int_{\Lambda^{\prime N} \nu \mid S_{a_{1}^{(N)}}^{(N)}} d x_{1} \ldots d x_{N} \\
\cdot \exp \left\{-\beta \sum_{1 \leqq i<j \leqq N} \tilde{v}\left(\left|x_{i}-x_{j}\right|\right)\right\} \cdot E_{N}(F) .
\end{gathered}
$$

Now, the components of $\alpha_{i}^{(k)}$ are independent, for $i=1, \ldots, N$ and $k=1, \ldots, v$.

If $i \in[1, N]$ and $k \in[1, v]$ are fixed and

$$
F_{i}^{(k)} \equiv\left\{\sup _{0 \leqq s \leqq 1}\left|\alpha_{i}^{(k)}(s)\right| \leqq \alpha\right\}
$$

then [24, Chap. 13.7, p. 29]:

$$
E\left(F_{i}^{k}\right)=1-e^{-2 \alpha^{2}}, \forall i \in[1, N], \forall k \in[1, v],
$$

where $E$ denotes expectation with respect to one-dimensional Brownian bridge. Inserting (III.39) into (III.37), we obtain

Write, now

$$
\begin{aligned}
Z_{\Lambda, \mathrm{MB}}(\beta) \geqq & \frac{1}{N !}(2 \pi \beta)^{-v N / 2} \int_{\Lambda^{\prime v N} \backslash S_{\alpha_{1}^{(N)}}^{(N)}} d x_{1} \ldots d x_{N} \\
& \cdot \exp \left[-\beta \sum_{1 \leqq i<j \leqq N} \tilde{v}\left(\left|x_{i}-x_{j}\right|\right)\right] \cdot\left(1-e^{\left.-\frac{2 \delta^{2} a_{1}^{2}}{4 \beta v}\right)^{v N}} .\right.
\end{aligned}
$$

$$
\begin{gathered}
\int_{\Lambda^{\prime} \nu N \backslash S_{a_{1}}^{(N)}} d x_{1} \ldots d x_{N} \exp \left[-\beta \sum_{1 \leqq i<j \leqq N} \tilde{v}\left(\left|x_{i}-x_{j}\right|\right)\right]=\left(\int_{\Lambda^{\prime} N \backslash \backslash S_{a_{1}}^{(N)}} d x_{1} \ldots d x_{N}\right) \cdot I_{N}, \\
I_{N} \equiv \frac{\int_{\Lambda^{\prime N \nu} \backslash S_{a_{1}}^{(N)}} d x_{1} \ldots \ldots d x_{N} \exp \left[-\beta \sum_{1 \leqq i<j \leqq N} \tilde{v}\left(\left|x_{i}-x_{j}\right|\right)\right]}{\int_{\Lambda^{\prime} \nu S_{a_{1}}^{(N)}} d x_{1} \ldots d x_{N}}
\end{gathered}
$$


By (III.41 b), (III.26) and Fisher's method [21, Appendix A], already used in the proof of Theorem III.2, it is easy to obtain the bound

$$
\frac{1}{V_{A}} \log I_{N} \geqq-\beta \varrho D p\left(a_{1}\right),
$$

where $D$ is the constant in (III.26) and $p(\cdot)$ is a function of the hard-core diameter $a$, regular in the neighbourhood of any $a \neq 0$; in particular:

$$
p\left(a_{1}\right) \underset{a_{1} \rightarrow a \neq 0}{\longrightarrow} p(a) .
$$

Equations (III.40), (III.41), and (III.42) yield, together with definition (III.18):

$$
f_{\Lambda, \mathrm{MB}}(\beta) \geqq v \varrho \log \left(1-e^{-\frac{\delta^{2} a_{1}^{2}}{2 \beta v}}\right)-\beta \varrho D p\left(a_{1}\right)+f_{\Lambda^{\prime}, a_{1}}^{\mathrm{Cl}}(\beta)-v \varrho / 2 \log (2 \pi \beta) .
$$

In (III.44),

$$
f_{\Lambda^{\prime}, a_{1}}^{\mathrm{Cl}}(\beta)=\frac{1}{V_{\Lambda}} \log Z_{\Lambda^{\prime}, a_{1}}^{\mathrm{Cl}}(\beta),
$$

where $Z_{\Lambda^{\prime}, a_{1}}^{\mathrm{Cl}}(\beta)$ is the classical partition function of pure hard cores in region $\Lambda^{\prime}$, without the kinetic term, which we separated out as the last term in (III.44). Performing the same splitting of (III.41) for the classical partition function, using the positivity of $\tilde{v}$ and again separating out the kinetic term, we find:

$$
f_{\Lambda}^{\mathrm{Cl}}(\beta) \leqq f_{\Lambda, a}^{\mathrm{Cl}}+\frac{\nu \varrho}{2} \log (2 \pi \beta),
$$

where $f_{\Lambda, a}^{\mathrm{Cl}}$ denotes the classical free energy of pure hard cores of diameter $a$. By (III.44) and (III.45) we find in the thermodynamic limit [noticing that by (III.35) $f_{\Lambda^{\prime}, a_{1}}^{\mathrm{Cl}}$ has the same thermodynamic limit $f_{a_{1}}^{\mathrm{Cl}}$ as $\left.f_{\Lambda, a_{1}}^{\mathrm{Cl}}\right]$ :

$$
\Delta f(v, \beta) \geqq \frac{v}{v} \log \left(1-e^{-\frac{\delta^{2} a_{1}^{2}}{2 \beta v}}\right)+f_{a_{1}}^{\mathrm{Cl}}-f_{a}^{\mathrm{Cl}}-\beta \cdot \varrho \cdot D p\left(a_{1}\right) .
$$

III.2.2. The Bound (I.10) for $v=1$. Let now $0<\varepsilon<\frac{1}{2}, \beta \in\left[0, \beta_{0}\right]$, for some fixed $\beta_{0}$ sufficiently small, and

$$
\delta=\delta_{\beta}=\beta^{\frac{1}{2}-\varepsilon}
$$

in (III.46) $\left[\right.$ more properly, $\delta=\left(\frac{\lambda}{a}\right)^{1-2 \varepsilon}$, where $\lambda=\hbar \sqrt{\beta}$ is the thermal wavelength $]$. Then the first term in (III.46) is still $O\left(\exp \left(-\beta^{2 \varepsilon}\right)\right)$, for $\beta$ sufficiently small. Since $a_{1}=a_{1}(\beta)=\frac{a}{1-\delta_{\beta}}$ by (III.34b), then (III.43) implies that the term $\left(-\beta \varrho D p\left(a_{1}\right)\right)$ in (III.46) is of order $O(\beta)$. To see what happens with

$$
\Delta f^{\mathrm{Cl}}(\beta) \equiv f_{a_{1}(\beta)}^{\mathrm{Cl}}-f_{a}^{\mathrm{Cl}}
$$

consider $v=1$, where [25]

$$
f_{a}^{\mathrm{Cl}}=\varrho[1+\log (v-a)] .
$$


We shall assume that

$$
v \geqq \xi a_{1}(\beta), \forall \beta \in\left[0, \beta_{0}\right]
$$

for some fixed $\xi$ satisfying

$$
1<\xi<\frac{3}{2}
$$

(Clearly $v>a_{1}(\beta)=\frac{a}{1-\delta_{\beta}}$ is necessary for $f_{a_{1}(\beta)}^{c}$ to be defined.) On $\delta_{\beta}$ we impose

$$
\delta_{\beta} \leqq 2(\xi-1) \text { for all } \beta \in\left[0, \beta_{0}\right] .
$$

Then, by (III.48) and (III.49):

$$
\Delta f^{\mathrm{Cl}}(\beta)=\varrho \log \left[1-\frac{\delta_{\beta} \cdot a}{\left(1-\delta_{\beta}\right)(v-a)}\right],
$$

with

$$
\frac{\delta_{\beta} a}{\left(1-\delta_{\beta}\right)(v-a)} \leqq \frac{\delta_{\beta}}{(\xi-1)+\delta_{\beta}} \leqq \frac{2}{3} .
$$

Using now the inequality

$$
\log (1-x) \geqq-\alpha x \quad \text { if } \quad x \leqq 1-\frac{1}{\alpha}, \quad(\alpha>1)
$$

with $\alpha=3$, we obtain

$$
\Delta f^{\mathrm{Cl}}(\beta) \geqq-3 \varrho \frac{\delta_{\beta} a}{\left(1-\delta_{\beta}\right)(v-a)} \geqq-\frac{3 a \varrho}{(3-2 \xi)(v-a)} \delta_{\beta},
$$

if, by (III.50) and (III.52):

$$
v \geqq \frac{\xi}{3-2 \xi} a
$$

Clearly, by (III.54), we may choose $v$ arbitrarily close to a (from above) by choosing $\xi$ arbitrarily close to one, but the interval of variation of $\beta$ shrinks in correspondence, as (III.52) shows. We have thus proved

Proposition III.1. Given $\xi$ satisfying (III.51) and $0<\varepsilon<\frac{1}{2}$, then (I.10) holds for $v=1$ if $(\beta, v)$ is such that $\beta \in\left[0, \beta_{0}\right]$, where $\beta_{0} \leqq 2^{\frac{2}{(1-2 \varepsilon)}}(\xi-1)^{\frac{2}{(1-2 \varepsilon)}}$, and $v$ satisfies (III.54).

Notice that (III.47) shows that an integrable potential outside the hard core provides a contribution to the free energy of order $O\left(\beta^{1 / 2}\right)$ with respect to the hard core term. This agrees with the intuition that for $\beta \downarrow 0$ only the behaviour of the potential near the origin plays a role - see also the introduction.

III.2.3. Lower Bound for $\Delta f(v, \beta)$ Using Coherent States. Assumption (III.27) of monotone decrease of the potential excludes several interesting cases, for instance, potentials which are negative (attractive) at large distances. In this section we 
sketch a derivation of the weaker bound (I.11), which is valid, however, for any stable hard-core potential, based on inequalities due to Baumgartner [5, Sect. 2c]. The latter involve an ingenious form of the coherent states which was introduced by Thirring [26]. Since there is no novelty in method, we shall be rather cursory in exposition, referring the reader to [5] for details. The present application reveals, however, the flexibility of the Thirring coherent states, when the wave-function is appropriately chosen.

We assume a two-body interaction (for clarity in this section we use a vector notation):

$$
V\left(\mathbf{x}_{1}, \ldots, \mathbf{x}_{N}\right)=\sum_{1 \leqq i<j \leqq N} v\left(\left|\mathbf{x}_{i}-\mathbf{x}_{j}\right|\right),
$$

with

$$
v(|\mathbf{x}|)=\left\{\begin{array}{lll}
\infty & \text { if } & |\mathbf{x}| \leqq a \\
\tilde{v}(|\mathbf{x}|) & \text { if } & |\mathbf{x}|>a
\end{array},\right.
$$

where $\tilde{v}$ is such that $V$ is thermodynamically stable. As in Sect. (III.2) $\tilde{v}$ will not play any significant role for small $\beta$. Let $v=3$ (just to agree with [5]). Then $[5,(25)$ et seq. $\left[Z_{\Lambda}(\beta)\right.$ stands for $Z_{\Lambda, \mathrm{B}}(\beta)$ or $\left.Z_{\Lambda, \mathrm{F}}(\beta)\right]$ :

$$
Z_{\Lambda}(\beta) \geqq \frac{1}{N !} \int_{\Lambda^{3 N \backslash S_{a}^{(N)}}} \frac{d^{3 N} p d^{3} x}{(2 \pi \hbar)^{3 N}} \exp [-\beta \hat{H}(\mathbf{p}, \mathbf{x})],
$$

where

$$
\begin{aligned}
\hat{H}(\mathbf{p}, \mathbf{x})= & \sum_{i=1}^{N}\left(\mathbf{p}_{i}^{2}+2 \mathbf{p}_{i} \hbar \cdot\langle\phi \mid \mathbf{p} \phi\rangle+\hbar^{2}\left\langle\phi \mid \mathbf{p}^{2} \phi\right\rangle\right) \\
& +\sum_{1 \leqq i<j \leqq N} \hat{v}\left(\left|\mathbf{x}_{i}-\mathbf{x}_{j}\right|\right)
\end{aligned}
$$

and

$$
\hat{v}(|\mathbf{x}|)=\left\{\begin{array}{ll}
\int d^{3} z d^{3} y v(|\mathbf{x}-\mathbf{y}|)|\phi(\mathbf{z}-\mathbf{y})|^{2}|\phi(-\mathbf{z})|^{2}, & |\mathbf{x}|>a \\
\infty, & |\mathbf{x}| \leqq a
\end{array} .\right.
$$

Above, $\phi$ is a $C^{\infty}$ function of compact support supp $\phi$ satisfying

$$
\text { and }
$$$$
\text { a) } \int|\phi(\mathbf{x})|^{2} d^{3} x=1 \text { (normalization) }
$$

b) $\operatorname{supp} \phi \subseteq\left\{\mathbf{x} \in \mathbb{R}^{3} ;|\mathbf{x}|<a\right\}$

$$
\begin{aligned}
\left\langle\phi \mid \mathbf{p}^{2} \phi\right\rangle & \equiv \int d^{3} p|\tilde{\phi}(\mathbf{p})|^{2} \mathbf{p}^{2}, \\
\langle\phi \mid \mathbf{p} \phi\rangle & \equiv \int d^{3} p|\tilde{\phi}(\mathbf{p})|^{2} \mathbf{p},
\end{aligned}
$$

where $\tilde{\phi}$ denotes the Fourier transform of $\phi$. We choose the hard-core diameter (" $\ell$ " in [5]) equal to the hard-core diameter $a$ of the original potential. We were also rather informal in definitions such as (III.55) and (III.58), but mathematical details (involving suitable limiting procedures) may be filled in as in Sect. III.1. We now choose $\phi$ as follows. Let $h(x)$ be a $C^{\infty}$-function of one real variable, such that $\operatorname{supp} h \subseteq[-1,1], \int_{-\infty}^{\infty} d x h(x)=1$, and define for $0<\alpha<\frac{a}{\sqrt{3}}$,

$$
h_{\alpha}(x)=\frac{1}{\alpha} h\left(\frac{x}{\alpha}\right) \text {. }
$$


Then $h_{\alpha} \in C^{\infty}$, supp $h_{\alpha} \subseteq[-\alpha, \alpha]$ and $\int_{-\infty}^{\infty} d x h_{\alpha}(x)=1$. Let, now

$$
\phi(\mathbf{x})=\phi_{\alpha}(\mathbf{x})=\prod_{i=1}^{3} \sqrt{h_{\alpha}\left(x_{i}\right)}=\prod_{i=1}^{3} \frac{1}{\sqrt{\alpha}} \sqrt{h\left(\frac{x_{i}}{\alpha}\right)}, \mathbf{x}=\left(x_{i}\right)_{i=1}^{3} \text {. }
$$

We also assume that $\sqrt{h}$ is $C^{\infty}$. This is satisfied, for instance, by the standard example of a $C^{\infty}$ function of compact support

$$
h(x)=\left\{\begin{array}{ll}
c \exp \left(+\frac{1}{x^{2}-1}\right) & |x| \leqq 1 \\
0 & \text { otherwise }
\end{array}\left(\text { with } c \text { such that } \int_{-1}^{1} h(x) d x=1\right) .\right.
$$

Condition a) is clearly satisfied, and b) follows from $\alpha<\frac{a}{\sqrt{3}}$ assumed above. Further, since $\phi$ is $C^{\infty}$, both quantities (III.59) and (III.60) are finite. Since $\phi$ is realvalued, (III.60) shows that

$$
\langle\phi \mid \mathbf{p} \phi\rangle=\mathbf{0} .
$$

With the choices above, the function $\psi$ defined by

$$
\psi(\mathbf{y})=\int d^{3} z|\phi(\mathbf{z}-\mathbf{y})|^{2}|\phi(-\mathbf{z})|^{2}
$$

is of compact support, and

$$
\operatorname{supp} \psi \leqq\left\{\mathbf{y} \in \mathbb{R}^{3} ;|\mathbf{y}| \leqq 2 \sqrt{3} \alpha\right\} .
$$

Hence, by (III.55), (III.58), and (III.65),

$$
\hat{v}(|\mathbf{x}|)=\tilde{v}(|\mathbf{x}|) \quad \text { if } \quad|\mathbf{x}|>a_{1}=a+2 \sqrt{3} \alpha .
$$

By (III.56), (III.63), (III.66), and definition (III.18) [where $f_{\Lambda}(\beta)$ stands for either $f_{\Lambda, \mathrm{B}}(\beta)$ or $\left.f_{\Lambda, \mathrm{F}}(\beta)\right]$ :

$$
\begin{aligned}
f_{\Lambda}(\beta) \geqq & \varrho \log \int \frac{d^{3} p}{(2 \pi \hbar)^{3}} \exp \left(-\beta \mathbf{p}^{2}\right)-\beta \varrho \hbar^{2}\left\langle\phi \mid \mathbf{p}^{2} \Phi\right\rangle \\
& +\frac{1}{V_{\Lambda}} \log \frac{1}{N !} \int_{\Lambda^{3 N} \backslash S_{a_{1}^{(N)}}} d^{3 N} x \exp \left[-\beta \sum_{1 \leqq i<j \leqq N} \tilde{v}\left(\left|\mathbf{x}_{i}-\mathbf{x}_{j}\right|\right)\right] .
\end{aligned}
$$

Let, now

$$
S_{\alpha}(x) \equiv \sqrt{h_{\alpha}(x)}
$$

Then, by (III.61)

$$
\left\langle\phi \mid \mathbf{p}^{2} \phi\right\rangle=\frac{3}{2 \pi} \int_{-\infty}^{\infty} d p p^{2}\left|\tilde{S}_{\alpha}(p)\right|^{2},
$$


where

$$
\tilde{S}_{\alpha}(p)=\int_{-\infty}^{\infty} d x e^{i p x} \sqrt{h_{\alpha}(x)}=\sqrt{\alpha} \int_{-\infty}^{\infty} d x e^{i p \alpha x} \sqrt{h(x)} .
$$

Upon substituting this expression for $\tilde{S}_{\alpha}(p)$ into (III.68) one obtains

$$
\left\langle\phi \mid \mathbf{p}^{2} \phi\right\rangle=\frac{3}{2 \pi \alpha^{2}} \int_{-\infty}^{\infty} d p p^{2} \widetilde{\left(h^{1 / 2}\right)}(p)^{2}
$$

and hence

$$
\left\langle\phi \mid \mathbf{p}^{2} \phi\right\rangle \leqq c \alpha^{-2}
$$

We may now handle the third term in (III.67) exactly as in Sect. (III.2). The leading term in $\beta$ will be due to the difference $\Delta f^{\mathrm{Cl}}$ in classical free energies corresponding to hard core diameters $a_{1}$ and $a$. Assuming that the classical free energy for $v=3$ behaves as the one-dimensional case, i.e., like the right-hand side of (III.49); (for a discussion of this assumption in the context of mean-field models, see $[25$, p. 93])

$$
f_{a}^{\mathrm{Cl}}=\varrho\left[1+\log \left(v^{1 / 3}-a\right)\right]
$$

(with $\varrho=1 / v$ the three-dimensional density and $a$ the hard core radius as before), we have

$$
\begin{aligned}
\Delta f^{\mathrm{Cl}} & =\varrho\left[1+\log \left(v^{1 / 3}-a_{1}\right)\right]-\varrho\left[1+\log \left(v^{1 / 3}-a\right)\right] \\
& =\left(v^{1 / 3}>a_{1}>a\right)=\varrho \log \left[1-\left(a_{1}-a\right) /\left(v^{1 / 3}-a\right)\right] \\
& \geqq-3 \varrho\left(a_{1}-a\right) /\left(v^{1 / 3}-a\right),
\end{aligned}
$$

if $\left(a^{1}-a\right) /\left(v^{1 / 3}-a\right)<\frac{2}{3}$, as in Sect. III.2.2. Now, $a_{1}-a=2 \sqrt{3} \alpha$ by (III.66). Take

$$
\frac{\alpha}{v^{1 / 3}-a}=\alpha^{-2} \beta \hbar^{2} \Rightarrow \alpha=\left(\beta \hbar^{2}\right)^{1 / 3}\left(v^{1 / 3}-a\right)^{1 / 3} \text {. }
$$

Then

$$
\frac{a_{1}-a}{v^{1 / 3}-a}=2 \sqrt{3} \frac{\left(\beta \hbar^{2}\right)^{1 / 3}\left(v^{1 / 3}-a\right)^{1 / 3}}{v^{1 / 3}-a}<\frac{2}{3}
$$

which may be written in terms of the thermal wave-length $\lambda=\sqrt{\pi \beta} \hbar$ :

$$
\left(\lambda / \sqrt{\pi}\left(v^{1 / 3}-a\right)\right)^{2 / 3}<1 /(3 \sqrt{3})
$$

or $\lambda<\frac{\sqrt{\pi}}{(3 \sqrt{3})^{3 / 2}}\left(v^{1 / 3}-a\right)$. Under this condition

$$
\Delta f^{\mathrm{Cl}} \geqq-3 \varrho \frac{2 \sqrt{3} \alpha}{v^{1 / 3}-a}=-3 \varrho 2 \sqrt{3} \frac{(\lambda / \sqrt{\pi})^{2 / 3}}{\left(v^{1 / 3}-a\right)^{2 / 3}},
$$

which is of the form (I.11). 


\section{Appendix A}

In this appendix, we collect some lemmas used in the main text.

\section{Lemma A.1.}

$$
\operatorname{tr}\left(f_{n}\left(e^{-\beta H}-e^{-\beta H_{0}}\right) f_{n}\right) \underset{n \rightarrow \infty}{\longrightarrow} \operatorname{tr}\left(e^{-\beta H}-e^{-\beta H_{0}}\right) .
$$

Proof. It suffices to show the following general result. If $A$ is a trace-class operator and $\left\{B_{n}\right\}$ a uniformly bounded sequence of operators converging weakly to $B$, then

$$
\lim _{n} \operatorname{tr}\left[A\left(B_{n}-B\right)\right]=0 .
$$

Let $\left\{e_{p}\right\}$ denote a complete orthonormal set of eigenvectors of $A^{*} A$ with eigenvalues $\left\{\lambda_{p}\right\}, \lambda_{p} \geqq 0$, and let $f_{p} \equiv \lambda_{p}^{-1 / 2} A e_{p}$ for $\lambda_{p} \neq 0$. Then $\sum \lambda_{p}^{1 / 2}=\|A\|_{\mathrm{tr}}<\infty$ and

$$
\operatorname{tr}\left[A\left(B_{n}-B\right)\right]=\sum_{p} \lambda_{p}^{1 / 2}\left(e_{p},\left(B_{n}-B\right) f_{p}\right)
$$

By the uniform boundedness of $B_{n}$, the sum over $p>p_{0}$ is bounded by

$$
\left(\sup _{n}\left\|B_{n}-B\right\|\right) \sum_{p>p_{0}} \lambda_{p}^{1 / 2}
$$

which can be made arbitrarily small for a suitable choice of $p_{0}$. The finite sum over $p \leqq p_{0}$ for any fixed $p_{0}$ tends to zero by the weak convergence $B_{n}-B \rightarrow 0$.

Lemma A.2. Let $v \in L^{\infty}\left(\Lambda^{v N}\right)$ and $\left\{U_{m}\right\}_{m \geqq 0}$ be the set of interactions on $\mathscr{H}_{\Lambda}^{(N)}$ defined in (III.7). Then $\left(H_{0, \Lambda}+U_{m}+V\right)$ is self-adjoint on $D\left(H_{0, \Lambda}\right)$ and converges to the Friedrichs extension of $\left(H_{0, \Lambda}^{a}+V\right)$ in the strong resolvent sense, i.e., (III.8) holds.

Proof. The proof is almost identical to the proof of Theorem 1.6, p. 224, of [17]. Slight alterations due to the extra Dirichlet condition on $\partial \Lambda$ are straightforward. Assumption $v \neq 2$ of [27] is not required because under our assumption on $v$ the Friedrichs extension and the form sum extension are equal.

Lemma A.3. Assume (III.8). Then (III.15) holds, i.e.,

$$
\exp \left[-t\left(H_{0, \Lambda}+U_{m}+V\right)\right] \psi \underset{m \rightarrow \infty}{\longrightarrow} T_{t} \psi \quad \forall \psi \in \mathscr{H}_{\Lambda}^{(N)},
$$

uniformly in any finite interval of $t \geqq 0$ (in the main text we use $\beta$ for $t$ ).

Proof. By the assumption(III.5) et seq. both $\left(H_{0, \Lambda}+U_{m}+V\right)$ and $H_{\Lambda}^{a} \equiv$ Friedrichs extension of $\left(H_{0, \Lambda}^{a}+V\right)$ belong to $\mathscr{G}(M, \beta)$ for some fixed $M, \beta$ (independent of $m$ ), in the notation of [28, p. 485]. Hence Theorem 2.16, p. 502 of [28] applies to yield (A.2).

\section{Proposition A.1.}

$$
\operatorname{tr}_{\mathscr{H}\left({ }_{A}^{(N)}\right.}\left(P_{B} \exp \left[-\beta\left(H_{0, \Lambda}+U_{m}+V\right)\right]\right) \underset{m \rightarrow \infty}{\longrightarrow} \operatorname{tr}_{\mathscr{H}_{A}^{(N)}}\left(P_{B} \exp -\beta H_{A}^{a}\right), \quad(\beta>0)
$$

(see Sect. III.1). 
Proof. By a variation of Theorem 6.6 of [11] (the variation being for the restriction to a finite volume $\Lambda$ with the Dirichlet condition), the operator $\exp \left[-\beta\left(H_{0, \Lambda}+U_{m}+V\right)\right]$ has a continuous positive kernel which we denote by $K_{m}(x, y)$, where $x=\left(x_{1}, \ldots, x_{N}\right), \quad x_{j} \in \mathbb{R}^{v}$, and similarly for $y$. Hence $P_{B} \exp \left[-\beta\left(H_{0, A}+U_{m}+V\right)\right]$ has the kernel

$$
\widetilde{K}_{m}(x, y)=N !^{-1} \sum_{p} K(P x, y) .
$$

By the Feynman-Kac formula (in the proof of Theorem 6.6 of [11]) $K_{m}(x, y)$ and hence $\widetilde{K}_{m}(x, y)$ are monotone decreasing functions of $m$ for each fixed $x, y$. Therefore we have the following limits

$$
\begin{aligned}
& \lim _{m \rightarrow \infty} K_{m}(x, y)=K_{\infty}(x, y) \\
& \lim _{m \rightarrow \infty} \widetilde{K}_{m}(x, y)=\tilde{K}_{\infty}(x, y) .
\end{aligned}
$$

Since, by (III.15), $\exp \left[-\beta\left(H_{0, \Lambda}+U_{m}+V\right)\right]$ converges strongly to $T_{\beta}$, as $m \rightarrow \infty$, $K_{\infty}(x, y)$ is the kernel of $T_{\beta}$ and $\widetilde{K}_{\infty}(x, y)$ that of $P_{B} T_{\beta}$. If we establish the fact that $\exp \left[-\beta\left(H_{0, \Lambda}+U_{m}+V\right)\right]$ and $\exp \left(-T_{\beta}\right)$ are in the trace class, then we can use the lemma, p. 65 of $[20]$ to obtain

$$
\begin{gathered}
\operatorname{tr} P_{B} \exp \left[-\beta\left(H_{0, A}+U_{m}+V\right)\right]=\int \widetilde{K}_{m}(x, x) d x, \\
\operatorname{tr} P_{B} \exp \left(-T_{\beta}\right)=\int \widetilde{K}_{\infty}(x, x) d x,
\end{gathered}
$$

and therefore

$$
\lim _{m \rightarrow \infty} \operatorname{tr} P_{B} \exp \left[-\beta\left(H_{0, \Lambda}+U_{m}+V\right)\right]=\operatorname{tr} P_{B} \exp \left(-T_{\beta}\right),
$$

which is (A.3). In order to prove that the above operators are trace class, we may use a variant of Theorem 21.4 of [11] and reduce the problem to the case without potential and without hard core. For a cube $\Lambda, \exp \left(-\beta H_{0,1}\right)$ is trace class by exact computation.

\section{Appendix B}

In this appendix we show that $\Delta f(\beta) \underset{\beta \downarrow 0}{\sim} \beta$, where $\Delta f(\beta)$ is, for purposes of comparison, the same quantity defined in (I.1 a), in the case of quantum spin systems. This follows from a trivial application of Lieb's inequalities [3]:

$$
Z_{\Lambda}^{\mathrm{Cl}}(S) \leqq Z_{\Lambda}^{\mathrm{Q}}(S) \leqq Z_{\Lambda}^{\mathrm{Cl}}(S+1)
$$

(with the same notation as [3]). In order to avoid unnecessary notational complications, assume that

$$
H_{\Lambda}^{\mathrm{Q}}=\sum_{x, y \in \Lambda} J(x-y) \mathbf{S}_{x} \cdot \mathbf{S}_{y},
$$

with

$$
\sum_{x \in \mathbb{Z}^{v}}|J(x)|<\infty \text { (stability) }
$$


and $\mathbf{S}$ spin operators corresponding to spin quantum number $S$. Then (B.1) and (B.2) together imply

$$
\begin{aligned}
f_{\Lambda}^{\mathrm{Cl}}(\beta, S)-f_{\Lambda}^{\mathrm{Cl}}(\beta, 1) & \leqq f_{\Lambda}^{\mathrm{Q}}(\beta, S)-f_{\Lambda}^{\mathrm{Cl}}(\beta, 1) \equiv \Delta f(\beta) \\
& \leqq f_{\Lambda}^{\mathrm{Cl}}(\beta, S+1)-f_{\Lambda}^{\mathrm{Cl}}(\beta, 1),
\end{aligned}
$$

where $f_{\Lambda}^{\mathrm{Q}}(\beta, S)$ [respectively $\left.f_{\Lambda}^{\mathrm{Cl}}(\beta, S)\right]$ is the quantum (respectively classical) free energy per particle, defined as in (III.18). The latter corresponds to the classical Hamiltonian

$$
H_{\Lambda}^{\mathrm{Cl}}=\sum_{x, y \in \Lambda} J(x-y)\left(S \mathbf{t}_{x} \cdot S \mathbf{t}_{y}\right),
$$

where $\mathbf{t}_{x}$ are three-dimensional unit vectors. From (B.3) we obtain immediately, in the thermodynamic limit

$$
-c \beta\left|S^{2}-1\right| \leqq \Delta f(\beta) \leqq c \beta\left[(S+1)^{2}-1\right],
$$

where $c \equiv \sum_{x \in \mathbb{Z}^{v}}|J(x)|$.

\section{Appendix C}

In this appendix we show that the high energy limit of the microcanonical entropy of an infinite system of particles in a bounded external potential is classical, by using the technique of Dirichlet-Neumann bracketing [10, Chap. XIII.15]. However, by performing the thermodynamic limit before the high-energy limit, we shall see that the corresponding estimates are quite different in character from those of [10, Chap. XIII.15]. We assume that

$$
\begin{gathered}
H_{\Lambda}^{D}=\sum_{i=1}^{N} h_{i}, \\
h_{i}=-\Delta_{i}^{D}+v\left(\mathbf{x}_{i}\right), \\
|v(\mathbf{x})| \leqq \alpha \text { for some } \alpha<\infty,
\end{gathered}
$$

where $\Delta^{D}$ is the Laplacian with Dirichlet boundary conditions on a cube $\Lambda=[-a, a]^{3}$ with volume $V=(2 a)^{3}$. We let

$$
\begin{aligned}
& \mu(E, V, N)=\frac{1}{N !}\left(\text { \# eigenvalues of } H_{\Lambda}^{D} \leqq E\right), \\
& g(E, V, N)=\frac{\tau_{3 N}}{(2 \pi \hbar)^{3 N} N !} \int_{\mathbf{x} \in \Lambda ; v(\mathbf{x}) \leqq E} d^{3 N} x\left(E-v_{T}(\mathbf{x})\right)^{3 N / 2},
\end{aligned}
$$

where $\tau_{3 N}$ is the volume of the unit sphere in $3 N$-dimensions, and

$$
v_{T}(\mathbf{x}) \equiv \sum_{i=1}^{N} v\left(\mathbf{x}_{i}\right)
$$

Let, also

$$
\begin{gathered}
\varrho=N / V, \\
v_{+, T}(N) \equiv \sup _{\mathbf{x} \in \Lambda^{N}} v_{T}(\mathbf{x})=N v_{\max },
\end{gathered}
$$


and

$$
v_{-, T}(N)=\inf _{\mathbf{x} \in \Lambda^{N}} v_{T}(\mathbf{x})=N v_{\min },
$$

where, by (C.2)

$$
\begin{aligned}
& v_{\text {max }} \leqq \alpha, \\
& v_{\text {min }} \geqq-\alpha .
\end{aligned}
$$

Let

$$
\begin{aligned}
g_{ \pm}(E, V, N) & =\frac{\tau_{3 N}}{(2 \pi \hbar)^{3 N} N !} \underset{\mathbf{x} \in \Lambda ; v_{ \pm}^{T} \leqq E}{ } d^{3 N} x\left(E-v_{ \pm}(N)\right)^{3 N / 2}, \\
S(E, V, N) & \equiv \frac{1}{V} \log \mu(E, V, N), \\
\mathrm{S}_{\mathrm{Cl}, \pm}(E, V, N) & =\frac{1}{V} \log g_{ \pm}(E, V, N), \\
S_{\mathrm{Cl}}(E, V, N) & =\frac{1}{V} \log g(E, V, N), \\
\mu_{+}(E, V, N) & =\frac{1}{N !}\left(\# \text { eigenvalues of } H_{+}^{D} \leqq E\right), \\
\mu_{-}(E, V, N) & =\frac{1}{N !}\left(\# \text { eigenvalues of } H_{-}^{N} \leqq E\right),
\end{aligned}
$$

where

$$
\begin{aligned}
& H_{+}^{D} \equiv-\Delta^{D}+v_{+, T}(N) ;-\Delta^{D} \equiv \sum_{i=1}^{N}\left(-\Delta_{i}^{D}\right), \\
& H_{-}^{N} \equiv-\Delta^{N}+v_{-, T}(N) ;-\Delta^{N}=\sum_{i=1}^{N}\left(-\Delta_{i}^{N}\right),
\end{aligned}
$$

and $\Delta_{i}^{D}$ (respectively $\Delta_{i}^{N}$ ) are the Laplacians with Dirichlet (respectively Neumann) boundary conditions (defined as in [10, Chap. XIII.15]). Finally, let

$$
S_{ \pm}(E, V, N)=\frac{1}{V} \log \mu_{ \pm}(E, V, N) .
$$

\section{Proposition C.1.}

$$
\begin{aligned}
& \lim _{\varepsilon \rightarrow \infty} \limsup _{\substack{N \rightarrow \infty \\
V \rightarrow \infty \\
N / V=\varrho}}\left[(V \varepsilon, V, N)-S_{\mathrm{Cl}}(V \varepsilon, V, N)\right] \\
& =\lim _{\varepsilon \rightarrow \infty} \liminf _{\substack{N \rightarrow \infty \\
V \rightarrow \infty \\
N / V=\varrho}}\left[S(V \varepsilon, V, N)-S_{\mathrm{Cl}}(V \varepsilon, V, N)\right]=0 .
\end{aligned}
$$

Proof. We have, by Dirichlet-Neumann bracketing [10, Chap. XIII.15] and (C.11):

$$
\mu_{+}(E, V, N) \leqq \mu(E, V, N) \leqq \mu_{-}(E, V, N) .
$$


Let $\varepsilon>\varrho_{\alpha}$. We have

$$
\begin{gathered}
\tau_{3 N}=\frac{\pi^{3 N / 2}}{\Gamma\left(\frac{3 N}{2}+1\right)}=\left(\frac{3 N}{2}\right)^{-\frac{3 N}{2}} \frac{(\pi e)^{3 N / 2}}{\sqrt{3 \pi N}\left(1+o\left(N^{-1}\right)\right)}=\left(\frac{3 N}{2}\right)^{-\frac{3 N}{2}} c_{N},(\text { C.16) } \\
c_{N}=\frac{(\pi e)^{3 N / 2}}{\sqrt{3 \pi N}\left(1+o\left(N^{-1}\right)\right)}
\end{gathered}
$$

We may therefore write, by (C.7):

$$
\sigma(\varepsilon, V, N) \leqq g_{+}(E, V, N) \leqq g(E, V, N) \leqq g_{-}(E, V, N) \leqq \tau(\varepsilon, V, N),
$$

where

$$
\begin{aligned}
& \sigma(\varepsilon, V, N)=\frac{1}{N !}\left[\frac{V}{(2 \pi \hbar)^{3}}\right]^{N} \exp \left[\frac{3 N}{2} \log \left(\frac{2 V}{3 N}\right)\right](\varepsilon-\varrho \alpha)^{\frac{3 N}{2}} c_{N}, \\
& \tau(\varepsilon, V, N)=\frac{1}{N !}\left[\frac{V}{(2 \pi \hbar)^{3}}\right]^{N} \exp \left[\frac{3 N}{2} \log \left(\frac{2 V}{3 N}\right)\right](\varepsilon+\varrho \alpha)^{\frac{3 N}{2}} c_{N} .
\end{aligned}
$$

By (C.15) and (C.18)

$$
\frac{\mu_{+}(E, V, N)}{g_{-}(E, V, N)} \leqq \frac{\mu(E, V, N)}{g(E, V, N)} \leqq \frac{\mu_{-}(E, V, N)}{g_{+}(E, V, N)}
$$

Now

$$
\frac{\mu_{+}(E, V, N)}{g_{-}(E, V, N)}=\frac{\mu_{+}(E, V, N) / g_{+}(E, V, N)}{1+\frac{g_{-}(E, V, N)-g_{+}(E, V, N)}{g_{+}(E, V, N)}},
$$

and hence, by (C.10a), (C.13), and (C.21) with $E=V \varepsilon$ :

$$
\begin{aligned}
\frac{1}{V} \log \frac{\mu_{+}(E, V, N)}{g_{-}(E, V, N)}= & S_{+}(E, V, N)-S_{\mathrm{Cl},+}(E, V, N) \\
& -\frac{1}{V} \log \left[1+\frac{g_{-}(E, V, N)-g_{+}(E, V, N)}{g_{+}(E, V, N)}\right] .
\end{aligned}
$$

By (C.8) and (C.2)

$$
0 \leqq g_{-}(E, V, N)-g_{+}(E, V, N) \leqq \frac{3 N}{2} \frac{\tau_{3 N}}{(2 \pi \hbar)^{3 N} N !} V^{\frac{3 N}{2}-1} \cdot V^{N}(\varepsilon+\varrho \alpha)^{\frac{3 N}{2}-1}(2 N \alpha),
$$

and therefore

$$
\begin{aligned}
0 & \leqq \frac{g_{-}(E, V, N)-g_{+}(E, V, N)}{g_{+}(E, V, N)} \leqq 3 N^{2} \alpha V^{-1}\left(\frac{\varepsilon+\varrho \alpha}{\varepsilon-\varrho \alpha}\right)^{\frac{3 N}{2}-1}(\varepsilon-\varrho \alpha)^{-1} \\
& =3 \varrho \alpha N\left(\frac{\varepsilon+\varrho \alpha}{\varepsilon-\varrho \alpha}\right)^{\frac{3 N}{2}-1}(\varepsilon-\varrho \alpha)^{-1} .
\end{aligned}
$$


Hence, for sufficiently large $N$

$$
\begin{aligned}
& 1+\frac{g_{-}-g_{+}}{g_{+}} \leqq 6 \varrho \alpha N\left(\frac{\varepsilon+\varrho \alpha}{\varepsilon-\varrho \alpha}\right)^{\frac{3 N}{2}-1}(\varepsilon-\varrho \alpha)^{-1} \\
& \frac{1}{V} \log \left(1+\frac{g_{-}-g_{+}}{g_{+}}\right) \leqq \frac{1}{V} \log \left[6 \varrho \alpha N(\varepsilon-\varrho \alpha)^{-1}\right] \\
&+\left(\frac{3 N}{2}-1\right) / V \log \left(\frac{\varepsilon+\varrho \alpha}{\varepsilon-\varrho \beta}\right) .
\end{aligned}
$$

By (C.20)-(C.23),

$$
\begin{aligned}
& S(E, V, N)-S_{\mathrm{Cl}}(E, V, N) \geqq S_{+}(E, V, N)-S_{\mathrm{Cl},+}(E, V, N) \\
& -\frac{1}{V} \log \left[6 \varrho \alpha N(\varepsilon-\varrho \alpha)^{-1}\right]-\left(\frac{3}{2} \varrho-\frac{1}{V}\right) \log \left(\frac{\varepsilon+\varrho \alpha}{\varepsilon-\varrho \alpha}\right) .
\end{aligned}
$$

Similarly, from (C.20),

$$
\begin{aligned}
& S(E, V, N)-S_{\mathrm{Cl}}(E, V, N) \leqq S_{-}(E, V, N)-S_{\mathrm{Cl},-}(E, V, N) \\
& +\frac{1}{V} \log \frac{g-(E, V, N)}{g_{+}(E, V, N)} \leqq S_{-}(E, V, N)-S_{\mathrm{Cl},-}(E, V, N) \\
& \quad+\frac{3}{2} \varrho \log \frac{\varepsilon+\varrho \alpha}{\varepsilon-\varrho \alpha}+\frac{1}{V} \log \left[6 \varrho \alpha N(\varepsilon-\varrho \alpha)^{-1}\right]
\end{aligned}
$$

by (C.18) and (C.19). that

By (C.24) and (C.25) we shall have proven Proposition C.1 if we can establish

$$
\begin{aligned}
& \lim _{\substack{N \rightarrow \infty \\
V \rightarrow \infty \\
N / V=\varrho}} \frac{1}{V} \log \frac{\mu_{ \pm}(V \varepsilon, V, N)}{g_{ \pm}(V \varepsilon, V, N)}=0 . \\
& \varepsilon \text { arbitrary. }
\end{aligned}
$$

Proof of (C.26). a) Dirichlet-case (+):

$N ! \mu_{+}$is the number of unit cubes with "right upper" corner inside the sphere $S_{r}, r=r_{0} N^{5 / 6}$ ("Dirichlet-cubes").

$N ! g_{+}$is the volume of $S_{r}$ :

$$
N ! g_{+}=\frac{\tau_{3 N}}{h^{3 N}} r^{3 N} .
$$

Let $d_{0}=\sqrt{3 N}$ be the diagonal of a unit cube, then the sphere $S_{r-d_{0}}$ is completely filled with Dirichlet-cubes. Therefore

$$
\begin{gathered}
N ! \mu_{+} \geqq \frac{\tau_{3 N}}{h^{3 N}}\left(r-d_{0}\right)^{3 N} \\
1 \leqq \frac{g_{+}}{\mu_{+}} \leqq\left(1-\frac{d_{0}}{r}\right)^{-3 N}=\left(1-\frac{\sqrt{3}}{r_{0}} \frac{3^{1 / 3}}{(3 N)^{1 / 3}}\right)^{-3 N} \leqq e^{a N^{2 / 3}},
\end{gathered}
$$


with a suitable constant $a$. This implies

$$
\frac{1}{V} \log \frac{g_{+}}{\mu_{+}} \leqq a \frac{N^{2 / 3}}{V} \underset{\substack{N \rightarrow \infty \\ V \rightarrow \infty \\ N / V=\varrho}}{\longrightarrow} 0 .
$$

b) Neumann-case (-):

$N ! \mu_{-}$is the number of unit cubes with "left lower" corner inside the sphere $S_{r}$. As above

$$
\begin{gathered}
N ! \mu_{-} \leqq \frac{\tau_{3 N}}{h^{3 N}}\left(r+d_{0}\right)^{3 N}, \\
1 \leqq \frac{\mu_{-}}{g_{-}} \leqq\left(1+\frac{d_{0}}{r}\right)^{3 N} \leqq e^{a^{\prime} N^{2 / 3}} .
\end{gathered}
$$

Acknowledgements. We should like to thank J. L. van Hemmen for reading the manuscript and suggesting some substantial improvements in Sect. II, and Prof. J. Ginibre for correspondence informing us about the present status of the problem. We are particularly indebted to the referee for important corrections and contributions to technical details, in particular Lemma A.1 and Proposition A.1 of Appendix A.

\section{References}

1. Fröhlich, J., Israel, R.B., Lieb, E.H., Simon, B.: Phase transitions and reflection positivity. I. General theory and long range lattice models. Commun. Math. Phys. 62, 1 (1978)

2. van Hemmen, J.L., Brito, A.A.S., Wreszinski, W.F.: Spin waves in quantum ferromagnets. J. Stat. Phys. 37, 187 (1984)

3. Lieb, E.H.: The classical limit of quantum spin systems. Commun. Math. Phys. 31, 327 (1973)

4. Simon, B.: The classical limit of quantum partition functions. Commun. Math. Phys. 71, 247 (1980)

5. Baumgartner, B.: Classical bounds on quantum partition functions. Commun. Math. Phys. 75, $25(1980)$

6. Griffiths, R.B.: Microcanonical ensemble in quantum statistical mechanics. J. Math. Phys. 6, 1447 (1965)

7. Minlos, R.A., Povzner, A.Ja.: Thermodynamic limit for entropy. Mat. Sb. 73, 269 (1967)

8. Driessler, W., Landau, L., Perez, J.F.: Estimates of critical lengths and critical temperatures for classical and quantum lattice systems. J. Stat. Phys. 20, 123 (1979)

9. Combe, Ph., Rodriguez, R., Sirugue, M., Sirugue-Collin, M.: Pub. Res. Inst. Math. Sci. 19, 355 (1983)

10. Reed, M., Simon, B.: Methods of modern mathematical physics, Vol. IV. Analysis of operators. New York: Academic Press 1978

11. Simon, B.: Functional integration and quantum physics. New York: Academic Press 1969

12. Jancovici, B.: Quantum-mechanical equation of state of a hard-sphere gas at high temperature. Phys. Rev. 178, 295 (1969). See also Jancovici, B., Merkuriev, S.P.: Quantummechanical third virial coefficient of a hard-sphere gas at high temperature. Phys. Rev. A 12, 2610 (1975) and references given there

13. Gibson, W.: Quantum-mechanical second virial coefficient at high temperatures. Phys. Rev. A 2, 996 (1970)

14. Huang, K.: Statistical mechanics. New York: Wiley 1963, Chap. 10.2

15. Calogero, F.: Variable phase approach to potential scattering. New York: Academic Press 1967, Chap. 15 
16. Ginibre, J.: Les Houches Lectures 1970. In: Mecanique statistique et theorie des champs. Stora, R., de Witt, C.M. (eds.). London: Gordon and Breach 1971

17. Lieb, E.H.: Calculation of exchange second virial coefficient of a hard-sphere gas by path integrals. J. Math. Phys. 8, 43 (1967)

18. Lebowitz, J.L., Penrose, O.: Analytic and clustering properties of thermodynamic functions and distribution functions for classical lattice and continuum systems. Commun. Math. Phys. 11, 99 (1968)

19. Ruelle, D.: Statistical mechanics. Rigorous results. New York: Benjamin 1969

20. Reed, M., Simon, B.: Methods of modern mathematical physics, Vol. III. Scattering theory. New York: Academic Press 1979

21. Fisher, M.E.: Arch. Ration. Mech. Anal. 17, 377 (1964)

22. Fricker, F.: Einführung in die Gitterpunktlehre. Basel: Birkhäuser 1982

23. Bratelli, O., Robinson, D.W.: Operator algebras and quantum statistical mechanics, Vol. II. Berlin, Heidelberg, New York: Springer 1981

24. Breiman, L.: Probability. Reading, MA: Addison Wesley 1968

25. Thompson, C.J.: Mathematical statistical mechanics. Princeton, NJ: Princeton University Press 1972

26. Thirring, W.: Lehrbuch der Mathematischen Physik, Vol. 4. Berlin, Heidelberg, New York: Springer 1980, p. 57

27. Ferrero, P., de Pazzis, O., Robinson, D.W.: Scattering theory with singular potentials. II. The $n$-body problem and hard cores. Ann. Inst. Henri Poincaré 21 A, 217 (1974)

28. Kato, T.: Perturbation theory for linear operators. 1 st ed. Berlin, Heidelberg, New York: Springer 1966

Communicated by M. E. Fisher

Received January 29, 1986; in revised form November 4, 1986 
